\title{
Comparison of Proactive Braking Intervention System Acceptability via Field Operation Tests in Different Regions
}

\author{
Takuma Ito ${ }^{1}$ - Ryosuke Matsumi ${ }^{1} \cdot$ Yuichi Saito $^{2} \cdot$ Akito Yamasaki $^{3} \cdot$ Shintaro Inoue $^{4} \cdot$ Tsukasa Shimizu $^{5}$. \\ Masao Nagai ${ }^{6} \cdot$ Hideo Inoue $^{7} \cdot$ Minoru Kamata $^{1}$
}

Received: 3 July 2020 / Revised: 6 August 2021 / Accepted: 23 September 2021 / Published online: 15 October 2021

(c) The Author(s) 2021

\begin{abstract}
To evaluate the generalities of acceptability of a proactive braking intervention system, which anticipates an imaginary pedestrian rushing out from occluded areas, and driving environmental dependencies, we conducted field operation tests (FOTs), where 146 elderly drivers participated at three evaluation sites with different urban characteristics. Analyses regarding the coexisting traffic participants illustrate driving environmental characteristics at each evaluation site. Evaluations via TMT-J, DSQ, and WSQ compare the characteristics of experimental participants. Based on these results as premises, we analyze questionnaire answers regarding the proposed system. As a result, we confirm the generalities that more than half of the participants at each evaluation site expressed a desire to introduce the proposed system into their own vehicle.
\end{abstract}

Keywords Proactive collision avoidance $\cdot$ ADAS $\cdot$ Elderly driver $\cdot$ FOT $\cdot$ Intelligent automobile $\cdot$ Community road

\section{Introduction}

In conjunction with the growth of the Japanese aged society [1], traffic accidents caused by elderly drivers have become a prominent social issue in Japan [2]. To help prevent such traffic accidents, we started the project to develop autonomous driving intelligence for supporting elderly drivers [3]. In support of these drivers, advanced driver assistance systems (ADAS) of which functions are automated to a certain

Takuma Ito

ito.t@hnl.t.u-tokyo.ac.jp

1 The University of Tokyo, 7-3-1 Hongo, Bunkyo, Tokyo, Japan

2 Tokyo University of Agriculture and Technology, 2-24-16 Nakacho, Koganei, Tokyo, Japan

3 Meijo University, 1-501 Shiogamaguchi, Tenpaku, Nagoya, Aichi, Japan

4 Toyota Motor Corporation, 1200 Mishuku, Susono, Shizuoka, Japan

5 Toyota Central R\&D Labs., Inc, 41-1 Yokomichi, Nagakute, Aichi, Japan

6 Japan Automotive Research Institute, 2530 Karima, Tsukuba, Ibaraki, Japan

7 Kanagawa Institute of Technology, 1030 Shimo-ogino, Atsugi, Kanagawa, Japan degree are desired to enhance the driving performance of elderly drivers which has usually deteriorated due to physical functional decline related to their aging [4]. To date, various driver assistance systems have been developed, such as Lane Keeping Assist (LKA), Adaptive Cruise Control (ACC), and Autonomous Emergency Braking (AEB) systems, for driving on major roads including highways. However, such driver assistance systems designed specifically for community roads, where elderly motorists drive cars daily, have not been sufficiently discussed.

As for the traffic accidents caused by elderly drivers on community roads in Japan, the Institute for Traffic Accident Research and Data Analysis (ITARDA) reported that collision accident at non-signalized crossings is one of typical cases [5]. Figure 1 shows an example of occluded crossings on Japanese community roads. As shown in the figure, visibility around non-signalized crossings on community roads is generally poor due to roadside structural occlusions such as house walls. Although pedestrians rushing out from such occluded areas are dangerous, AEB systems may not be effective due to the lack of time between their detection by on-board sensors and the conflict point arrival in such situations; thus, such pedestrians sometimes result in traffic accidents. Therefore, not only the event-based deceleration triggered by on-board sensors but also the proactive deceleration based on preliminary information are necessary for ADAS 


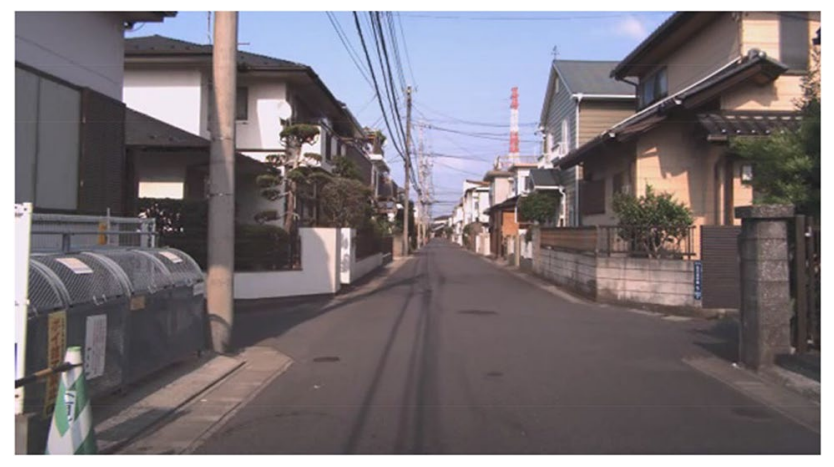

Fig. 1 Example of an occluded crossing

on community roads. On this point, because community roads are not usually equipped with roadside cooperative sensing systems such as the driving safety support system (DSSS) [6], anticipation of an imaginary pedestrian behind occluded areas is the key for proactive deceleration. Based on this concept, Saito et al. [7] developed proactive braking intervention systems with the foresighted driver model that anticipated a pedestrian rushing out from occluded areas around blind crossings.

For improving the new systems and familiarizing them to the public, evaluation of the acceptability of them is necessary. Although there are various methods to evaluate the acceptability, field operation tests (FOTs) by real target users using actual experimental vehicles on existing roads are important for not missing any consideration factors. In addition, although there are various driving environments in the real world, generalities of the acceptability and driving environmental dependencies have not been adequately discussed. Therefore, in this study, we intended to confirm the proactive braking intervention system acceptability via the FOT at more than one evaluation site for discussing generalities of the acceptability and driving environmental dependencies.

The remainder of this paper is organized as follows. Chapter 2 describes the proactive braking intervention system details and the acceptability evaluation strategy. The experimental details for evaluation are described in Chapter 3. The results and discussions are described in Chapter 4. Finally, Chapter 5 summarizes the conclusion and describes future work.

\section{Proactive Braking Intervention System}

\subsection{Concept of Proactive Braking Intervention System}

On community roads, pedestrians sometimes rush out from the occluded area around blind crossings. Although recent automobiles are sometimes equipped with AEB systems, detectable areas by on-board sensors may be partially occluded by surrounding roadside objects. In such situations, because AEB operational timing may be delayed from ideal one, it may result in shortage in deceleration of the vehicle. Furthermore, if the vehicle entry velocity into the crossings is high in such situations, collisions with pedestrians occur. Thus, proactive deceleration before operating AEB systems is important for compensating the on-board sensor limitations on community roads. Although mature human drivers usually conduct this kind of hazard-anticipatory driving, some drivers do not drive cars in that manner. Thus, an ADAS with the foresighted driver model that intervenes with driver operations and conducts proactive braking for the posterior AEB systems, in the event pedestrians actually do rush out, is desired. This is the proactive braking intervention system concept.

\subsection{Behavior of Proactive Braking Intervention System}

Figure 2 shows the targeted situational schematic of the proactive braking intervention system. Although the proactive braking intervention system needs to grasp the position of occluded crossings, the robust detection of such crossings by only the on-board sensors is difficult. Thus, our proposed system uses a digital map, which we call LeanMAP [8], as supplementary information. The LeanMAP consists of nodes and links for expressing the road network. In the LeanMAP system, the longitudinal vehicle position is expressed as a driving distance from an origin point of a link along the road. We call the longitudinal position "offset", as shown in Fig. 2. Moreover, the LeanMAP system contains crossing position information, which is expressed as offset. Furthermore, the localizer system can determine the vehicle position. Thus, our proposed system can estimate relative distance to blind crossings for the vehicle motion control. The motion control system based on the foresighted driver model developed by Saito et al. [7] assumes an imaginary pedestrian behind the occluded area around the blind crossing. As shown in Fig. 2, $D_{p}$ denotes an assumed distance between the imaginary pedestrian and a wall, $\mathrm{Y}_{\mathrm{p}}$ denotes an assumed distance between the imaginary pedestrian and the vehicle, $V_{p}$ denotes an assumed velocity of the imaginary pedestrian, $\mathrm{D}_{\text {car }}$ denotes the relative distance from the vehicle to the crossing, and $Y_{\text {gap }}$ denotes the distance between the vehicle and a wall. Based on some assumed parameters such as $\mathrm{D}_{\mathrm{p}}, \mathrm{Y}_{\mathrm{p}}$, and $\mathrm{V}_{\mathrm{p}}$, and other observable parameters such as $D_{\text {car }}$ and $Y_{\text {gap }}$, the proposed system calculates a reference velocity $\mathrm{V}_{\text {min }}$, which is a constant value depending on each situation, as a target velocity of proactive deceleration. The definition of $\mathrm{V}_{\min }$ is the maximum velocity at which the vehicle can avoid the collision with pedestrians on the 
Fig. 2 Targeted situational schematic of proactive braking intervention system

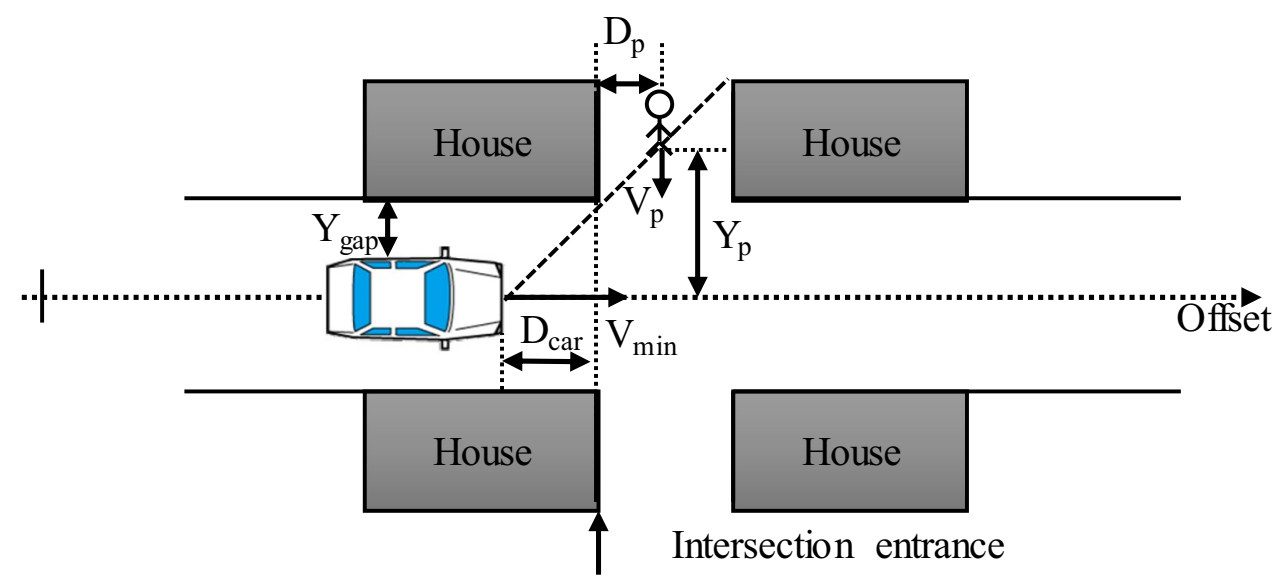

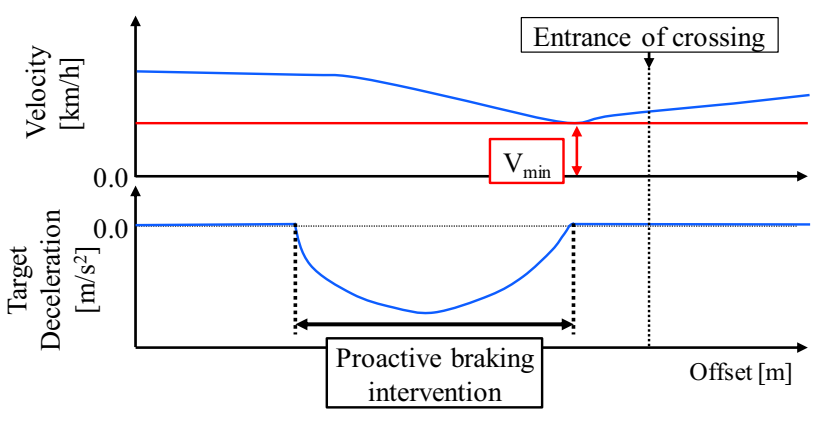

Fig. 3 Conceptual velocity profile schematic by proactive braking intervention

assumption of additional autonomous emergency braking systems in the target situation. Furthermore, the proposed system calculates the necessary deceleration value based on the $\mathrm{V}_{\min }$, time-series velocity, and relative distance to the crossings.

Figure 3 shows a conceptual velocity profile schematic by proactive braking intervention. The horizontal axis indicates offset. The vertical axis of the upper graph indicates the velocity, whereas that of the lower one indicates the target deceleration value calculated by the system. The upper graph blue line indicates the velocity profile, whereas the red line indicates the $\mathrm{V}_{\min }$ for a certain situation. If the vehicle velocity is greater than $\mathrm{V}_{\min }$ around a blind crossing, the system conducts the braking intervention for decelerating to $\mathrm{V}_{\text {min }}$ before the crossing entrance. The braking intervention end position is set slightly before the crossing entrance because the vehicle can detect the pedestrian, if he/she exists, at the position. The system starts braking intervention, if necessary, $5.0 \mathrm{~s}$ before passing through the end position. The timing of $5.0 \mathrm{~s}$ was determined in our previous study [9] to reproduce the deceleration of driving instructors, who were mature drivers. During the system intervention, the system ignores the driver acceleration operation. As for the motion control formulations, the details were described in the previous study [7].

Although the proposed system was primarily designed for proactive deceleration while approaching blind crossings, it can be used for proactive deceleration while overtaking parked cars that create occluded areas behind them. Although the digital map supplies the necessary information of relative distance for proactive deceleration around blind crossings, vehicle information detected by on-board sensors can supply similar information instead. Based on such information, the proposed system can calculate the equivalent $\mathrm{V}_{\text {min }}$ while overtaking parked cars. In addition, the proposed system can also be used for supporting drivers at stop lines. If 0.0 is assigned to $\mathrm{V}_{\min }$, the proposed system can stop the vehicle at the stop line, the position of which is registered in the LeanMAP. Because violating the regulation relating to stopping at stop lines sometime may cause traffic accidents on community roads, supporting elderly drivers in such situations seems useful. Thus, in this study, we tested not only proactive deceleration around the blind crossings but also the abovementioned functions.

Because the proposed system partially controls the vehicle independently from driver operations, the drivers may feel surprise and anxiety about such controls. To prevent this, the proposed system tells advanced notifications and current braking intervention states via a heads-up display (HUD). Figure 4 shows the conceptual transition schematic of the provided information. First, from $7.5 \mathrm{~s}$ before the end of braking intervention, the HUD beeps a sound and displays the proactive deceleration reason as an advance notification. Then, from $5.0 \mathrm{~s}$ before the end of braking intervention, the HUD beeps again and displays the current proactive deceleration states. The yellow side bar length decreases according to the remaining proactive braking intervention time. The displayed icons are different for each situation, as shown in the figure. The Japanese characters in the icons mean "stop line", "slow down" and "stop", respectively, as shown in 
Fig. 4 Conceptual transition schematic of provided HUD information

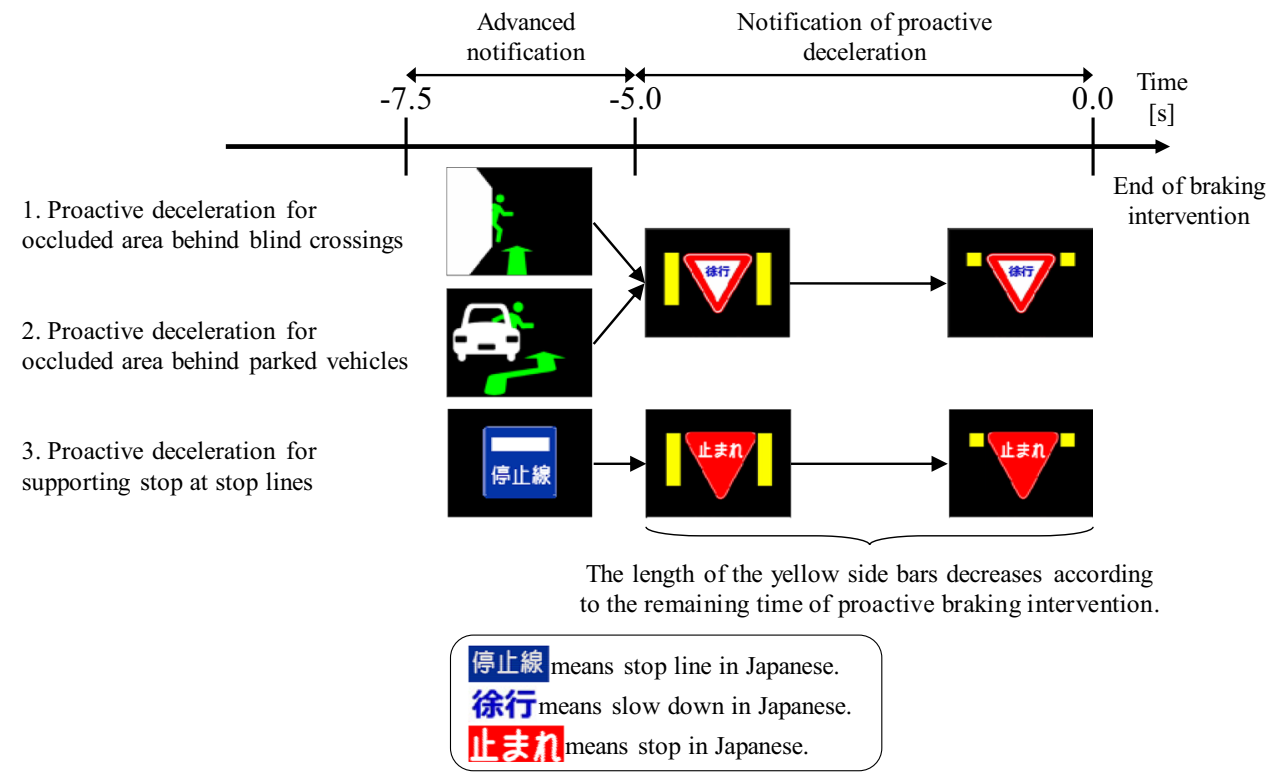

the figure. The details of designing the information sharing system are described in previous studies [10-12].

\subsection{Preparation of Data Collection for Evaluating Proactive Braking Intervention System}

To date, various FOTs have been conducted globally. One of the most famous FOTs was "euroFOT" [13], where 28 industrial companies and research institutes conducted large-scale FOTs for evaluating the ADASs in European countries. Their FOT activities consisted of 3 steps: Preparation, data collection, and analysis from various viewpoints. By following this architecture, we organized our project to evaluate the proposed system. Figure 5 shows the flowchart and summary of our previous and ongoing approaches for evaluating the proposed system. At the preparation stage, we conducted driving simulator (DS) experiments and two types of FOTs: Pre-FOT and Pilot FOT. First, we conducted DS experiments [7, 14] for extracting potential problems and summarizing the proposed system issues. Because the proposed system had new concepts of hazard anticipation and proactive braking intervention, we needed to observe the user behaviors in the controlled environments. Thus, we used DS as a first step. Then, we conducted Pre-FOTs [15, 16] for discussing the evaluation methods. The advanced points from DS experiments were that we conducted the evaluation tests in the real world by using actual experimental vehicles. However, because we did not know how the experimental participants would behave and interact with the proposed system, we conducted the Pre-FOT on the private test course for safety. After that, we conducted Pilot FOTs $[9,17]$ for confirming the unique problems on public roads. The advanced points from Pre-FOTs were that evaluations were conducted on public roads. Finally, based on the abovementioned preparation findings, we conducted Total FOT, which is described in this study, for collecting large-scale data at various sites. The advanced points from Pilot FOTs are that the data were collected at more than one region under basically the same experimental protocols for discussing the generalities of acceptability and variances depending on regional differences.

\subsection{Analyses of Collected Data}

There are various collected data analysis viewpoints for evaluating the proposed system. Because the primary objective of this study was the discussion regarding the generalities of acceptability of the proposed system and driving environmental dependencies, we needed to analyze both the proposed system acceptability and driving environmental characteristics at each evaluation site. However, when we collect the data for evaluation at multiple evaluation sites, not only do the driving environmental differences, but also the driver characteristics differences among the evaluation sites, if exists, may affect the proposed system evaluations. On this point, although we considered that there are naturally different driver characteristics among individual participants, the important point is whether or not there are group differences among evaluation sites. Thus, we also needed to confirm the driver characteristics of the experimental participants as premises for discussing the generalities and dependencies. In addition, because some parts of driver characteristics might be related to the urban characteristics and driving environmental characteristics, we needed to confirm the relations among them, if the differences of 


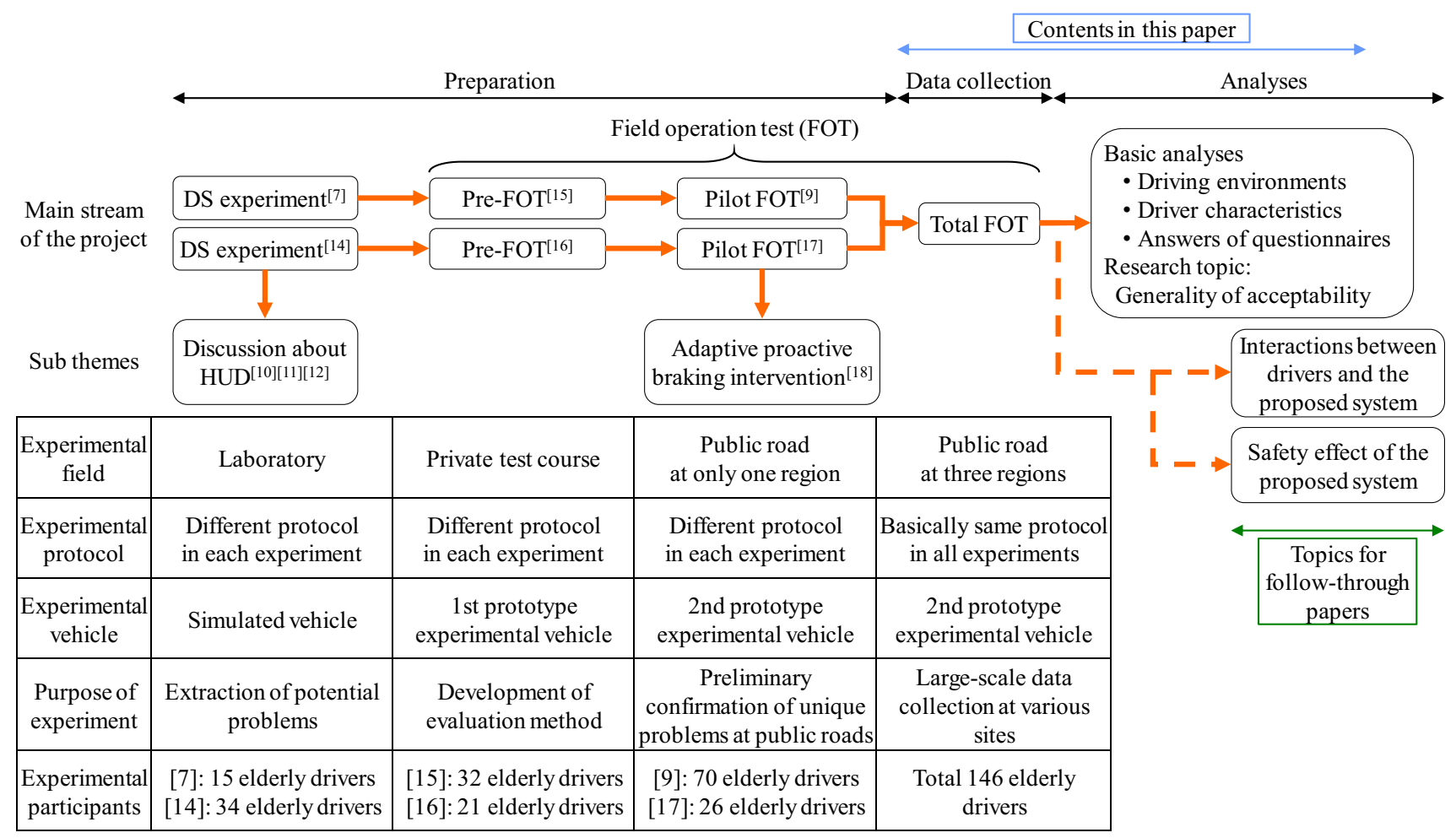

Fig. 5 Flowchart and summary of our approaches for evaluating the proactive braking intervention system

driver characteristics existed. Therefore, we investigate and analyze the following topics in this study.

- Driving environmental characteristics where data were collected

- Characteristics of experimental participants

- Proposed system acceptability via questionnaires

Although quantitative analyses from other viewpoints such as experimental participant behaviors and system effects for safe driving are also necessary for evaluating the proposed system in detail, the abovementioned topics require analysis prior to the other topics as a first step of followthrough analysis for our FOT data, as shown in Fig. 5.

\subsection{Acceptability Evaluation}

To date, acceptability of various in-vehicle driver assistance systems which are intervention type such as ACC and AEB has been examined in accordance with the development of such systems. Figure 6 shows the conceptual schematic of relation between driver assistance systems and driving situations. For example, ACC is usually used in relatively stable driving situations such as highway. Because drivers use such systems with their intentions, conflicts between the drivers and the systems basically do not occur. On the other hand, although AEB is executed independently of drivers' intentions, the situations are so dangerous that the drivers cannot do anything. Thus, conflicts between them basically do not occur also in this situation. However, the proactive braking intervention system is assumed to be used in the potentially dangerous situation. In this situation, the system interveners the vehicular motion controls prior to and independently of driver operations while the driver also intends to control the vehicle. Thus, conflicts between them may occur. To be more precise, the proposed system users may feel annoyance although they may simultaneously feel system benefits.

To discuss such complicated acceptability, in our previous study [14], we focused on the following three viewpoints as components of acceptability, and the viewpoint of desire
Fig. 6 Schematic of relation between driver assistance systems and driving situations (this figure is modified from one in our previous paper [14])

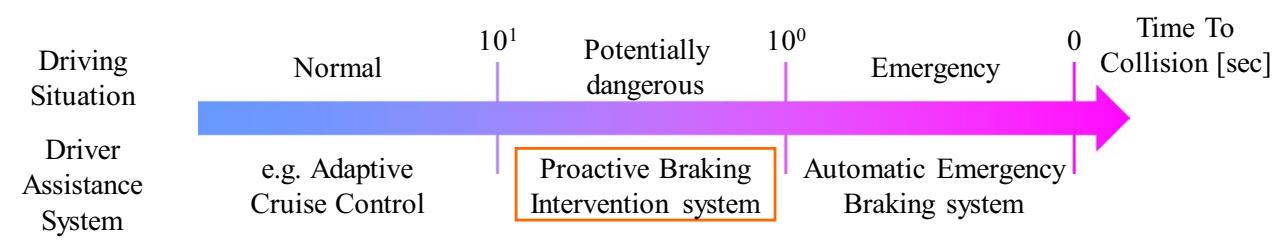


to introduce the proposed system into their own vehicles as an aspect of evaluating the total acceptability of the proposed system. Figure 7 shows the conceptual schematic of hierarchical sources of total acceptance.

- Reactive acceptance and refusal: Evaluation based on the instinctive factor during use of the system.

- Comprehensive acceptance and refusal: Evaluation based on sufficient understanding of the system merits and demerits.

- Reflective acceptance and refusal: Evaluation based on the self-image as the owner of the system among others and society.

In the previous studies $[14,16,17]$, we confirmed that the comprehensive factors were positively evaluated; further, it led to the positive evaluation of total system acceptance. However, such evaluations regarding each factor were conducted in the limited preparation stage conditions. In addition, because some parts of reactive factors might be sensitive to the driving environmental characteristics and driver characteristics, investigating reactive factors among different regions would be beneficial. Thus, in this study, we intended to discuss the generalities and differences of the evaluation results for each factor while considering regional differences. In other words, we strove to confirm whether or not evaluations of each factor depend on the regional differences.

\section{Experiments}

\subsection{Experimental Organization}

Experiments for data collection were conducted at three evaluation sites: The Koganei area conducted by Tokyo University of Agriculture and Technology, the Kashiwa area conducted by the University of Tokyo, and the Atsugi area conducted by Kanagawa Institute of Technology. The following protocols of each experiment for data collection and sharing of de-identified data among research collaborators

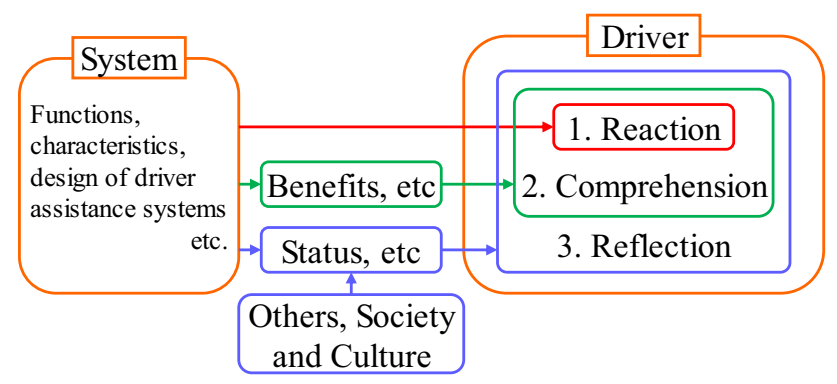

Fig. 7 Schematic of hierarchical acceptance sources [14] were approved by the institutional review board for human studies of the corresponding universities. We explained the experimental protocol to the participants, and obtained written informed consent from them.

\subsection{Experimental Participants}

Elderly drivers as experimental participants were recruited respectively by the research members of each experimental site. The following conditions were requested to them.

- They needed to have a valid driving licenses.

- They needed to drive a car regularly.

- They needed to be equal to or older than 65 years old.

As for the Koganei and Kashiwa areas, the experimental participants were dispatched from Silver Human Resources Centers at Koganei and Kashiwa. As a result, we recruited 67 participants from the Koganei area, and 65 participants from the Kashiwa area. Because they belonged to each Silver Human Resources Center, they lived in Koganei city and Kashiwa city. As for the Atsugi area, because we could not get them dispatched from Silver Human Resources Center, we recruited participants by the snowball sampling method. Although we recruited a total of 27 participants at the Atsugi area, some of them were retired workers of the company that participated in this project. Thus, because the analyses in this study focused primarily on the questionnaire answers that may have been affected by such relations with the participants, we excluded the data of those participants from the analyses regarding human factors to avoid biased data and a conflict of interest. As a result, we obtained valid data from 14 participants at the Atsugi area. In addition, because they were recruited by snowball sampling method, their residences were not limited to the city of Atsugi; however, they basically lived in the neighboring cities, where the driving environments were similar to those of Atsugi city. Table 1 shows the basic characteristics of experimental participants.

We paid honorarium to the experimental participants. For Koganei area, we paid the Silver Human Resources Centers at Koganei $1500 \mathrm{JPY} /$ hour, which is equivalent to approximately $14 \mathrm{USD} /$ hour at the rate of the that time, including administrative expenses, the rate of which was not open to us. For Kashiwa area, we paid the Silver Human Resources Centers at Kashiwa approximately 1500 JPY/hour excluding administrative expenses. For Atsugi area, we paid directly the experimental participants $1500 \mathrm{JPY} /$ hour. In addition, we also paid traveling expenses according to actual conditions. These honorariums were determined by the rules of each Silver Human Resources Center and each University. Although the honorarium for the experimental participants of Koganei area were bit smaller than those of other areas due to the administrative expenses of Silver Human Resources Center, 
Table 1 Basic characteristics of experimental participants

\begin{tabular}{llll}
\hline Experimental site & Koganei & Kashiwa & Atsugi \\
\hline Recruit method of participants & Dispatch from Silver Human Resources Centers & Snowball sampling \\
Number of participants & 67 & 65 & 14 \\
Male participants & 63 & 49 & 7 \\
Female Participants & 4 & 16 & 7 \\
Average age & 71.7 (SD: 4.6) & 72.2 (SD: 4.8) & 68.7 (SD: 4.7) \\
Number of participants in 65-69 & 23 & 22 & 10 \\
Number of participants in 70-79 & 41 & 39 & 4 \\
Number of participants in 80-89 & 3 & 4 & 0 \\
Average period for having driving licenses & 46.6 years (SD: 7.7) & 44.0 years (SD:12.4) \\
Average driving frequency per week & 2.4 days (SD: 1.5) & 3.9 days (SD: 1.8) & 47.3 years (SD: 7.2) \\
\hline
\end{tabular}

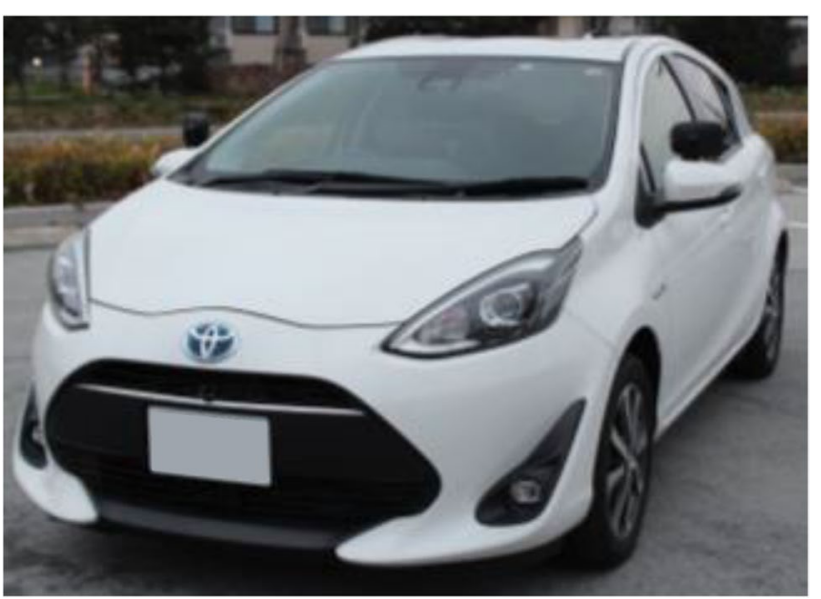

Fig. 8 Appearance of experimental vehicle

the differences among them were considered to be small. Thus, we considered that there were not any factors affecting the evaluations from the viewpoint of honorarium.

\subsection{Experimental Vehicle and Systems}

Figure 8 shows the appearance of the experimental vehicle. This vehicle was equipped with the proposed system discussed in Section 2.2. Thus, this vehicle could provide proactive braking interventions activated by the foresighted driver model, and display the information via HUD while approaching the target situations. Although the mathematical formula of longitudinal motion control algorithm was same as one proposed in the previous study [7], the parameters were bit updated. The details of the parameter determination were described in our other previous study [9]. Regarding the lateral control, because the system was incapable of maneuvering, the participants maneuvered the steering wheel by themselves. In addition, we logged the original information from control area network (CAN) and additional on-board sensors such as front cameras, LIDAR, GPS, and the midstream information processed by the system, such as digital map position, target control values, and so on. Almost equivalent vehicles were used in all three experimental sites.

\subsection{Experimental Course}

The main target situations of the proactive braking intervention system are non-signalized crossings with poor visibility due to roadside structural occlusions. Thus, although there are various driving environments in the real world, we selected some courses which have common characteristics on the above-mentioned point and different characteristics regarding other conditions such as types of coexisting traffic participants and traffic volume among the evaluation sites. Although the findings of this study would be limited by the selection of the evaluation sites, we thought that we could discuss the trends of acceptability and driving environmental dependencies to a certain degree. To be more precise, we collected the experimental data in three evaluation sites: The Koganei, Kashiwa, and Atsugi areas. Koganei is one of the cities in Tokyo, which is the capital of Japan. Because Koganei is very close to the center of Tokyo, Koganei has very tight residential areas. Kashiwa is one of cities in Chiba prefecture, which borders Tokyo. Because Kashiwa is also relatively close to the center of Tokyo, Kashiwa has the characteristics of Tokyo commuter towns. Therefore, Kashiwa has relatively tight residential areas. Atsugi is one of cities in Kanagawa prefecture, which also borders Tokyo. In contrast to Kashiwa, Atsugi is relatively distant from Tokyo. Thus, the city of Atsugi is sparser than the other two areas. As mentioned above, because the city characteristics seemed different from each other, we intended to collect the experimental data from such cities.

Figure 9 shows the experimental course in the Koganei area. This figure and following similar figures are based on the map images published by the Geospatial Information Authority of Japan [19], and we added some information, such as regulated speed, the links, and intervention 


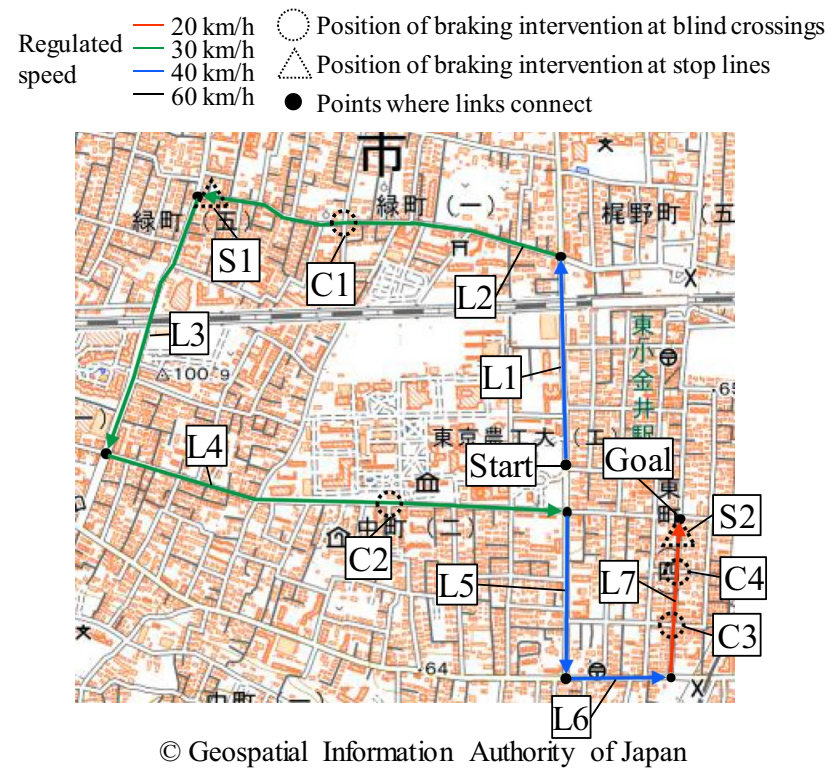

Fig. 9 Experimental course at Koganei area

positions. The orange areas indicate the positions of structures such as houses, buildings, and factories. This course consists of seven links labeled L1-L7. The link colors indicate the regulated speed; a red line for $20 \mathrm{~km} / \mathrm{h} \mathrm{limit,}$ green line for $30 \mathrm{~km} / \mathrm{h}$ limit, blue line for $40 \mathrm{~km} / \mathrm{h}$ limit, and black line for $60 \mathrm{~km} / \mathrm{h}$ limit, which is the maximum velocity on non-highway roads in Japan. The total course length is approximately $3.7 \mathrm{~km}$. We selected this course considering the following points.

- The course should contain several non-signalized crossings, and stop lines where the cars must stop.

- The time required to complete the course should be less than approximately $20 \mathrm{~min}$ for making the experimental participants not forget the details of each trial.

- The course should not contain excessively dangerous crossings.
- The course should reflect the characteristics of the evaluation area.

As for the fourth point, because Koganei area had the characteristics of very tight residential areas, we selected this course which included such characteristics. For the course selection of Kashiwa area and Atsugi area described in the following parts, we considered the above-mentioned points. Table 2 shows the characteristics of each link at the Koganei area. In this course, we set four crossings labeled $\mathrm{C} 1$ to $\mathrm{C} 4$ where the proactive braking interventions for blind crossings were activated, and two stop lines labeled S1 to S2 where those for stop lines were activated. We selected these intervention points considering the following points.

- The visibility around the intervention point should be poor.

- The risk of rea-end collision should be small when the system intervened the braking operation.

For the selection of intervention points in Kashiwa area and Atsugi area described in the following parts, we considered basically the same points. Table 3 shows the target $\mathrm{V}_{\text {min }}$ for each crossing. These values were determined considering the road width and the existence of road shoulders in each situation. In the Koganei area, the experiences of the proactive braking intervention while overtaking parked cars were not safe because parked cars were located at the relatively large roads where the traffic volume was relatively large. Thus, we considered that the experiments regarding the proactive braking intervention while overtaking parked cars were difficult in the Koganei area. Therefore, we did not

Table 3 Target $\mathrm{V}_{\min }$ for blind crossings at Koganei area

\begin{tabular}{lllll}
\hline Crossing No & C1 & C2 & C3 & C4 \\
\hline $\mathrm{V}_{\min }[\mathrm{km} / \mathrm{h}]$ & 7.2 & 18.6 & 5.9 & 5.9 \\
\hline
\end{tabular}

Table 2 Characteristics of each link at Koganei area

\begin{tabular}{lllll}
\hline Link No & Center Line & $\begin{array}{l}\text { Isolation with } \\
\text { pedestrian }\end{array}$ & Type of isolation & $\begin{array}{l}\text { Link } \\
\text { distance } \\
{[\mathrm{m}]}\end{array}$ \\
\hline L1 & Y & Y & Curb & 440 \\
L2 & N & Y & White line & 790 \\
L3 & Y & Y & Curb & 580 \\
L4 & N & Y & White line & 990 \\
L5 & Y & Y & Curve & 350 \\
L6 & Y & Y & White line or crash barrier & 220 \\
L7 & N (one-way road) & N & & 340 \\
\hline
\end{tabular}

"Y" means Yes. "N" means No 
set the areas for the proactive braking intervention around the parked cars, unlike the other evaluation sites.

Figure 10 shows the experimental course in the Kashiwa area. Because Kashiwa area had the characteristics of commuter towns, we selected this course which included such characteristics. This course consists of 12 links labeled L1-L12. The total course length is approximately $4.6 \mathrm{~km}$.
Table 4 shows the characteristics of each link in the Kashiwa area. In this course, we set seven crossings labeled C1-C7 where the proactive braking interventions for blind crossings were activated, and five stop lines labeled S1 to S5 where those for stop lines were activated. Table 5 shows the target $\mathrm{V}_{\min }$ for each crossing. Because some parts of L6, L8, and L11 were relatively safe situations, we set these areas for the
Fig. 10 Experimental course at Kashiwa area

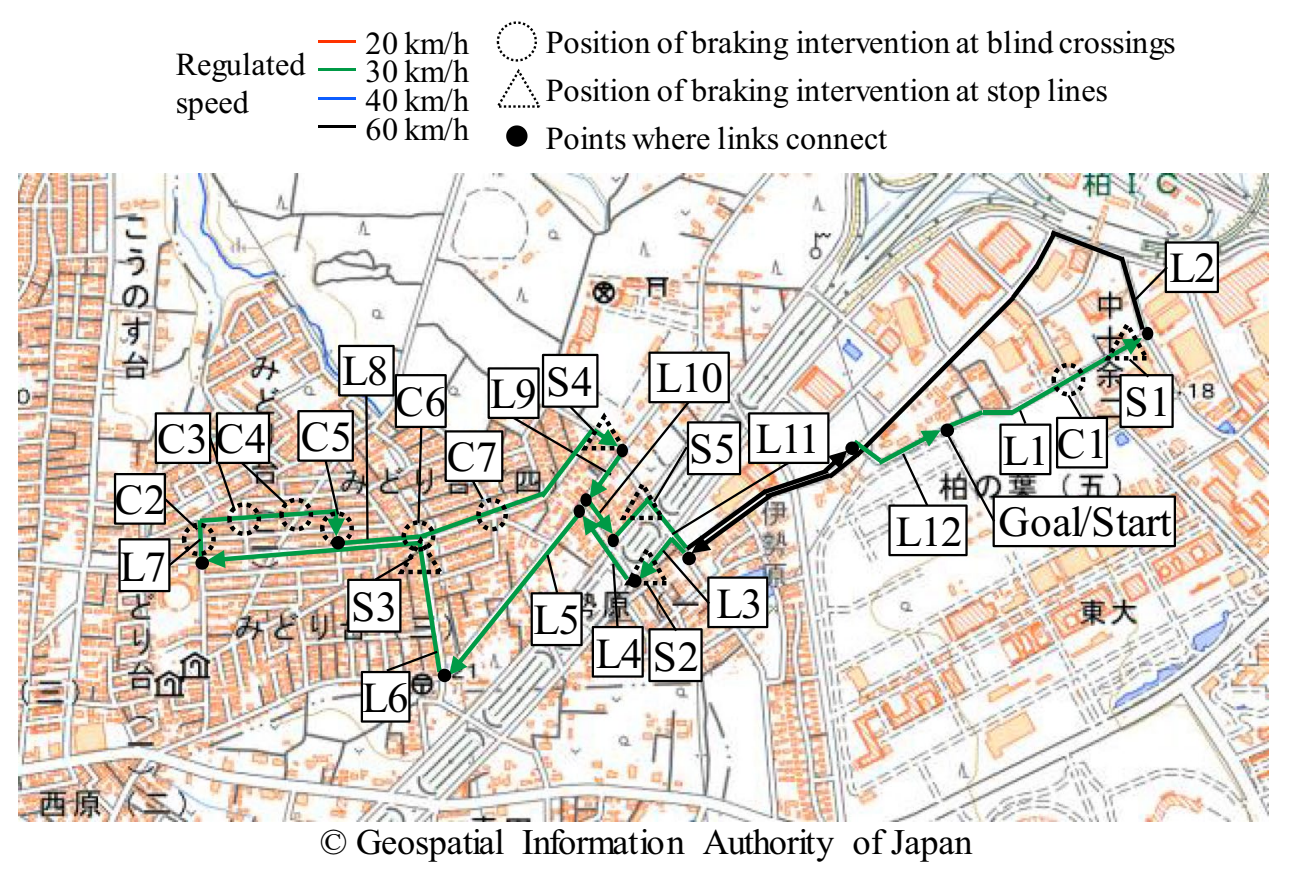

\begin{tabular}{llllr}
\hline Link No & Center Line & $\begin{array}{l}\text { Isolation with } \\
\text { pedestrian }\end{array}$ & Type of isolation & $\begin{array}{l}\text { Link } \\
\text { distance } \\
{[\mathrm{m}]}\end{array}$ \\
\hline L1 & Y & Y & White line or crash barrier & 450 \\
L2 & Y & Y & Curb & 1110 \\
L3 & N & Y & White line or crash barrier & 150 \\
L4 & N & Y & White line & 160 \\
L5 & N & Y & Crash barrier & 370 \\
L6 & Partly Y & Y & White line & 600 \\
L7 & N & N & & 350 \\
L8 & N & Y & White line or crash barrier & 560 \\
L9 & N & Y & Crash barrier & 120 \\
L10 & N & Y & White line & 90 \\
L11 & Y & Y & Curb & 570 \\
L12 & Y & Y & White line & 90 \\
\hline
\end{tabular}

"Y" means Yes. "N" means No

\begin{tabular}{llllllll}
\hline Crossing No & C1 & C2 & C3 & C4 & C5 & C6 & C7 \\
\hline $\mathrm{V}_{\text {min }}[\mathrm{km} / \mathrm{h}]$ & 23.7 & 4.7 & 4.7 & 4.7 & 4.7 & 22.9 & 21.1 \\
\hline
\end{tabular}

Table 5 Target $\mathrm{V}_{\min }$ for blind crossings at Kashiwa area
Table 4 Characteristics of each link at Kashiwa area 
proactive braking intervention while overtaking parked cars. To be more precise, if there was a parked car in these areas when the participants experienced the proposed system, it executed the proactive braking for the occluded areas due to the parked car. However, because we did not prepare the parked cars ourselves, the proposed system targeted only on the parked cars that were incidentally located in the areas. Thus, such proactive braking for a parked car was a rare case.

Figure 11 shows the experimental course in the Atsugi area. Because Atsugi area was sparser town than other two areas, we selected this course which included such characteristics. This course consists of 11 links labeled L1-L11. The total course length is approximately $6.1 \mathrm{~km}$. Table 6 shows the characteristics of each link at the Atsugi area. As for L3, $\mathrm{L} 4$, and L10, the regulated speed was $60 \mathrm{~km} / \mathrm{h}$ although they were not very large roads. This was not because high velocity was allowed due to the width of them, but because they were not regulated due to their triviality. In this course, we set four crossings labeled $\mathrm{C} 1-\mathrm{C} 4$ where the proactive braking interventions for blind crossings were activated, and two stop lines labeled S1 to S2 where those for stop lines were activated. Table 7 shows the target $\mathrm{V}_{\min }$ for each crossing. Similar to the Kashiwa area, we set the areas for the proactive braking intervention while overtaking parked cars in L4. However, because the situations where the other cars were parked in the area were very rare, the system rarely executed the proactive braking intervention for the parked cars.

\subsection{Experimental Procedure}

Figure 12 summarizes the experimental procedure flow. The main experimental parts were divided into two days for each participant. The Day 2 experiment was conducted 1 week after the Day 1 experiment. Before Day 1, participants answered preliminary questionnaires that asked about themselves. On Day 1, we first explained to the participants about the experiments and obtained their informed
Fig. 11 Experimental course at Atsugi area

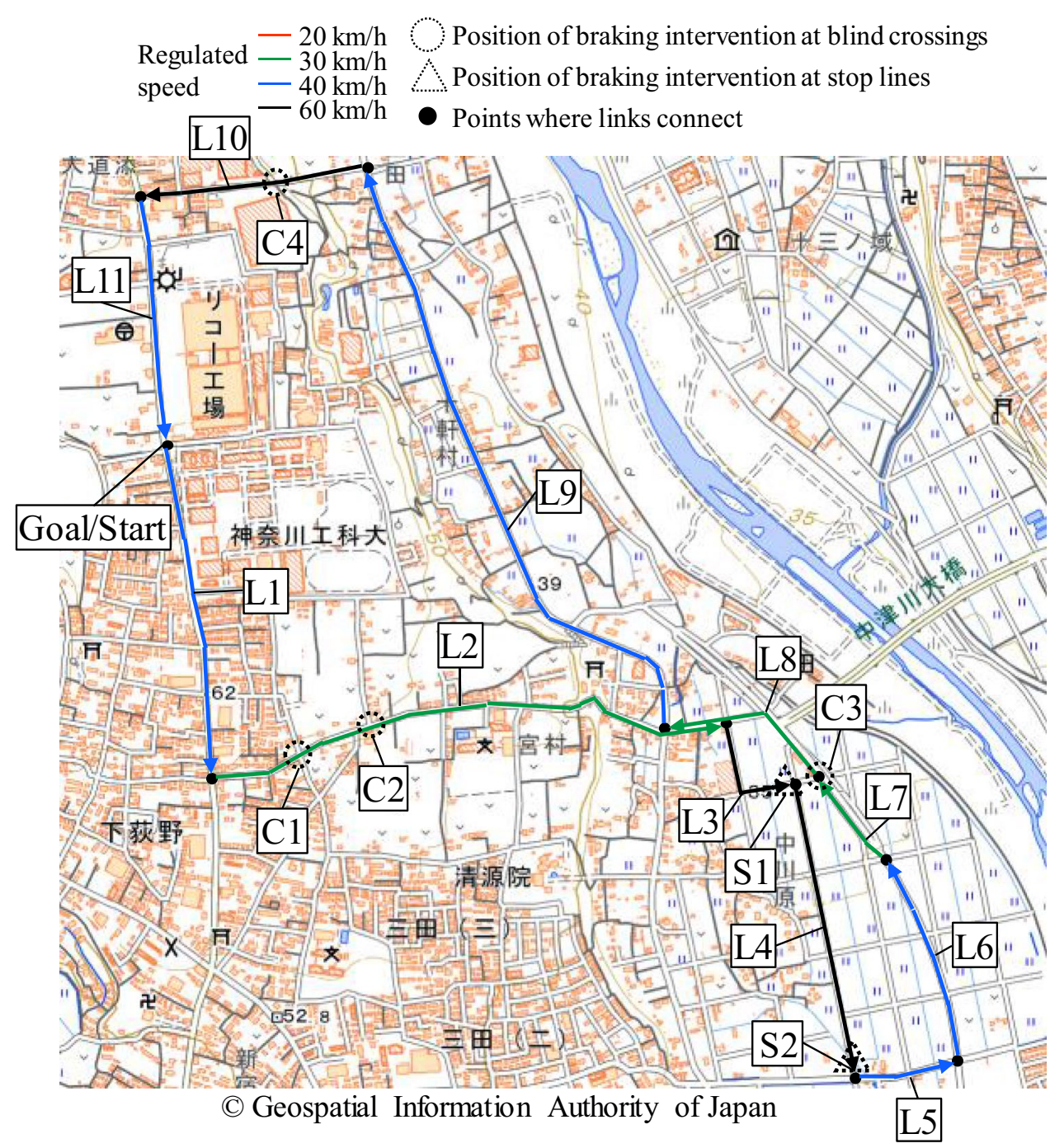


Table 6 Characteristics of each link at Atsugi area

\begin{tabular}{llllr}
\hline Link No & Center Line & $\begin{array}{l}\text { Isolation with } \\
\text { pedestrian }\end{array}$ & Type of isolation & $\begin{array}{l}\text { Link } \\
\text { distance } \\
{[\mathrm{m}]}\end{array}$ \\
\hline L1 & Y & Y & Curb & 680 \\
L2 & Y & Y & Crash barrier or curb & 1010 \\
L3 & N & N & & 260 \\
L4 & Y & Y & White line & 600 \\
L5 & Y & Y & White line & 220 \\
L6 & Y & Y & Curb & 450 \\
L7 & N (one-way road) & Y & Curb & 200 \\
L8 & Y & Y & Curb & 370 \\
L9 & Y & Y & Curb & 1340 \\
L10 & N & Y & White line or curb & 470 \\
L11 & Y & Y & Curb & 500 \\
\hline
\end{tabular}

"Y" means Yes. "N" means No
Table 7 Target $\mathrm{V}_{\min }$ for blind crossings at Atsugi area

\begin{tabular}{lllll}
\hline Crossing No & C1 & C2 & C3 & C4 \\
\hline $\mathrm{V}_{\min }[\mathrm{km} / \mathrm{h}]$ & 18.2 & 18.2 & 30.3 & 18.8
\end{tabular}

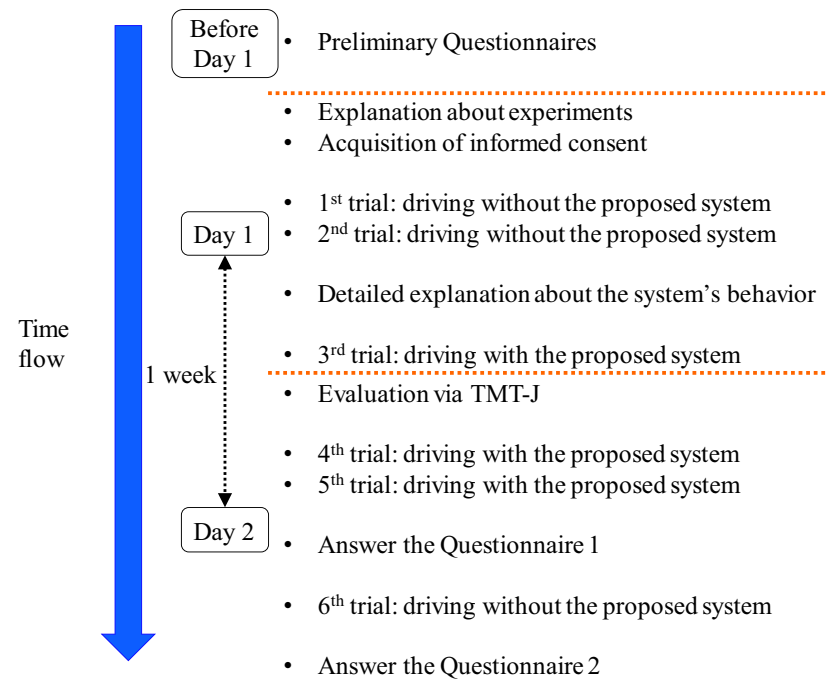

Fig. 12 Flow of experimental procedure

consent. Next, they drove the experimental vehicle twice on the course without the proposed system. Subsequently, we explained the details about the system behaviors during braking intervention. After that, they drove the vehicle once with the proposed system. On Day 2, they first answered the TMT-J which evaluated their ability regarding perception and recognition. The TMT-J details will be described in the following section. Next, they drove the experimental vehicle twice with the proposed system. Subsequently, they answered Questionnaire 1, which asked about the reactive factors of the system. We asked such questions at the time because we wanted them to recall the temporary feeling. After that, they drove the experimental vehicle once without the proposed system. Finally, they answered Questionnaires 2, which asked about the other factors of the proposed system.

\subsection{Experimental Instructions}

We gave the following instructions to the participants before the experiment.

- The participants will experience the two types of braking support system.

- The 1 st one operates at potentially dangerous situations around blind crossings and parked vehicles where pedestrians or cyclists might rush out from the occluded area. In such situations, the system decelerates to the desired velocity to maintain safety.

- The 2nd one operates at stop lines where the vehicle needs to stop. In such situations, the system decelerates to not overrun the stop line.

- The system function is not autonomous driving but only provides driving support to the participants. Thus, braking operations by the participants are also necessary.

- The participants can drive as they prefer although the participant should follow the traffic regulations.

\subsection{Questionnaires}

We used three types of questionnaires in this experiment. The first questionnaires were original ones which asked about the basic participant characteristics, such as age, 
(6)

(2)

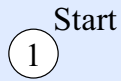

(5)

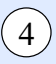

Fig. 13 Example of TMT-J Part A test sheet

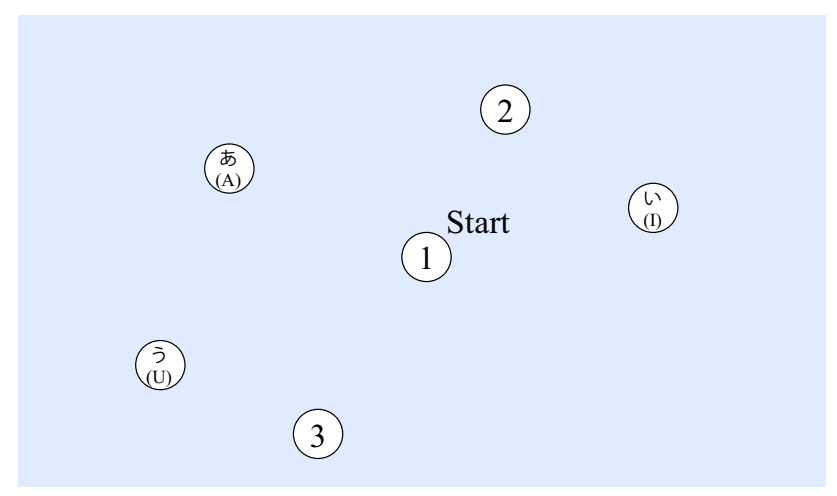

Fig. 14 Example of TMT-J Part B test sheet

average driving frequency, and so on. The main results of these questions are summarized in Table 1.

The second questionnaires were existing ones which evaluated the characteristics of the participants. To be more precise, we used TMT-J [20], which is a Japanese version of the Trail Making Test [21], for evaluating the total ability regarding perception and recognition, DSQ [22] for evaluating the driving style via 18 questions, and WSQ [23] for evaluating the workload sensitivity via 38 questions. DSQ and WSQ were tested before Day 1 as a preliminary questionnaire. The TMT-J consists of two parts: Part A and Part B. Figures 13 and 14 show the test sheet examples of Part A and Part B, respectively. These figures are only examples for explaining the tasks, and we used commercial ones in actuality. In the case of Part A, the participants draw lines connecting 25 circles with the numbers from 1 to 25 in a sequential order. In contrast, in the case of Part B, the participants draw lines connecting 13 circles with numbers and 12 circles with Japanese characters alternately. We measured the number of mistakes and the time to complete the task. Based on the combination of both criteria, we evaluated the ability of the participants.

The third questionnaires were original ones which asked about the various characteristics of the experienced system.
The topics were based on the previous studies findings $[14,16$, 17]. To be more precise, we asked about the following topics.

\section{Reactive factors}

To what degree did you feel interference with your driving from the support system at occluded crossings?

To what degree did you feel interference with your driving from the support system at stop lines?

To what degree did you feel anxiety for the support system at occluded crossings?

To what degree did you feel anxiety for the support system at stop lines?

To what degree did you feel strangeness for the support system at occluded crossings?

To what degree did you feel strangeness for the support system at stop lines?

Comprehensive factors

To what degree did you feel that the support system reduced your driving burden?

To what degree did you feel that you could drive safely owing to the support system?

\section{Reflective factors}

To what degree did you feel that the support system is necessary for elderly drivers in Japanese society?

To what degree did you feel that the support system is currently necessary for you?

To what degree did you feel that the support system would be necessary for you after five years?

Total acceptability

To what degree did you want to introduce the support system into your own vehicle?

The participants answered the questionnaire based on a grade scale of 1-6. The following shows the description of each grade.

- Grade 1: The participants felt very much.

- Grade 2: The participants felt so.

- Grade 3: The participants felt a little.

- Grade 4: The participants did not feel so much.

- Grade 5: The participants did not feel.

- Grade 6: The participants did not feel at all. 


\subsection{Statistical Analysis Methods}

In this study, we aimed to compare the results of questionnaires among evaluation sites. Thus, the number of comparison target was three. In addition, comparison targets were equivalent from each other, and there was no control group. Therefore, first, we compared the three groups for each analysis. Then, if the significant differences under the $5 \%$ significance level were confirmed, we conducted pairwise comparisons for confirming which pair had the significant differences. The detailed methods of these statistical analyses depend on the characteristics of questionnaires. The characteristics of questionnaires in this study were classified into three as follows form the viewpoints of statistical analysis methods.

1. Unpaired-samples comparison of non-parametric scales among three areas.

2. Unpaired-samples comparison of parametric scales among three areas.

3. Paired-samples comparison of non-parametric scales among three questionnaires.

Analyses of TMT-J and large part of original questionnaires were classified into the first case. In this case, we used the Kruskal-Wallis test as the comparison among three groups. Then, if we confirmed significant differences under the 5\% significance level, we used the Steel-Dwass test as pairwise comparisons. Analyses of DSQ and WSQ were classified into the second case. In this case, we used the one-way ANOVA as the comparison among three groups. Then, if we confirmed significant differences under the 5\% significance level, we used the Tukey-Kramer test as pairwise comparisons. The comparisons between some questionnaires, which will be described in the Section 4.3.2, were classified into the third case. In this case, we used the Friedman's test as the comparison among three groups. Then, if we confirmed significant differences under the 5\% significance level, we used the Steel-Dwass test as pairwise comparisons.

\section{Analyses}

\subsection{Analyses of Traffic Environments}

\subsubsection{Analysis Procedure}

To define the driving environmental characteristics of each evaluation site, we analyzed the time-series image data via the front camera of the experimental vehicle. Because our system targeted on accident prevention with pedestrians, coexisting pedestrians with the possibility of colliding with the vehicle are an important factor to discuss the system acceptability. In addition, because the proposed system intervenes with braking operations, which controls the velocity, traffic flow on the road is also an important factor. Thus, we visually analyze the collected data from the viewpoint of these factors.

As for the coexisting pedestrians, we visually counted the number of pedestrians which the experimental vehicle overtook or passed by. Because the pedestrians with no possibility of colliding with the vehicle were not important, we counted only those having a collision possibility, as shown in Fig. 15. As for the road with a center line, we counted only the pedestrians on the near side of the center line. As for the road without center lines, we counted the pedestrians on the both sides. As for the road with pedestrian isolation areas, we counted the pedestrians only in the area with road paint such as white lines, and not any area with physical barriers such as crash barriers and curbs.

As for the traffic flow, although a fixed-point observation to count the number of the vehicles on the experimental course was ideal, it needed too much additional data and took a lot of time and human resources. Thus, as a simplified method, we counted the number of oncoming vehicles instead.

Furthermore, because the weather conditions might affect the encounter ratio of the coexisting traffic participants, we analyzed only the data collected on sunny and cloudy days. As a result, the driving data numbers for analyzing the traffic environmental characteristics were 371 for the Koganei area, 308 for the Kashiwa area, and 139 for the Atsugi area. These data included the driving data for both with and without the proposed system. In addition, the aim of the analyses in this section was not the
Fig. 15 Rules for counting the coexisting pedestrians

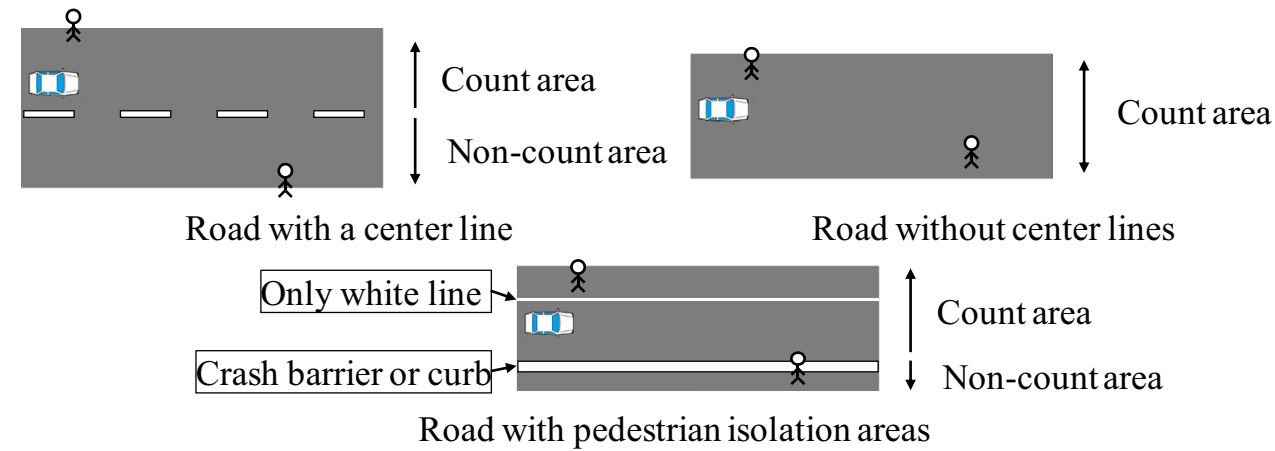

Road with pedestrian isolation areas 
participant evaluation but the traffic environmental evaluation. Thus, for the Atsugi area, because larger data numbers were better for the traffic environmental analyses, we analyzed not only the data from the 14 participants included in Section 3.2, but also the data from the 13 participants who were excluded from Section 3.2, for a total of 27 participants.

\subsubsection{Analysis Results at Each Evaluation Site}

Figure 16 shows the encounter ratios with pedestrians and oncoming vehicles in the Koganei area. Each mark with a number such as "L1" indicates the combination of the encounter ratios in the corresponding link. The circle symbols indicate the links where no braking intervention was conducted. The upward triangle symbols indicate the links where braking interventions were conducted at occluded crossings. The downward triangle symbols indicate the links where braking interventions were conducted at stop lines. The star-shaped symbols indicate the combination of upward and downward triangles, which indicate the links where braking interventions were conducted at both occluded crossings and stop lines. Although we wanted to express the results distributed in a wide range using the double logarithmic charts to grasp the general outlines, the numbers of coexisting pedestrians and oncoming vehicles were zero in some links. Because we could not express zero in the double logarithmic chart, we put such data on the axis for descriptive purposes. For example, as shown in Fig. 16, the encounter ratio with oncoming vehicles at L7 in the Koganei area was 0.0.

As for the results in the Koganei area, we confirmed two distributed groups. The Distribution A shown in the figure includes the results of L1, L3, L5, and L6. As shown in the

O: Links where no braking intervention was conducted.

$\triangle$ : Links where braking intervention was conducted at occluded crossings.

$\nabla:$ Links where braking intervention was conducted at stop lines.

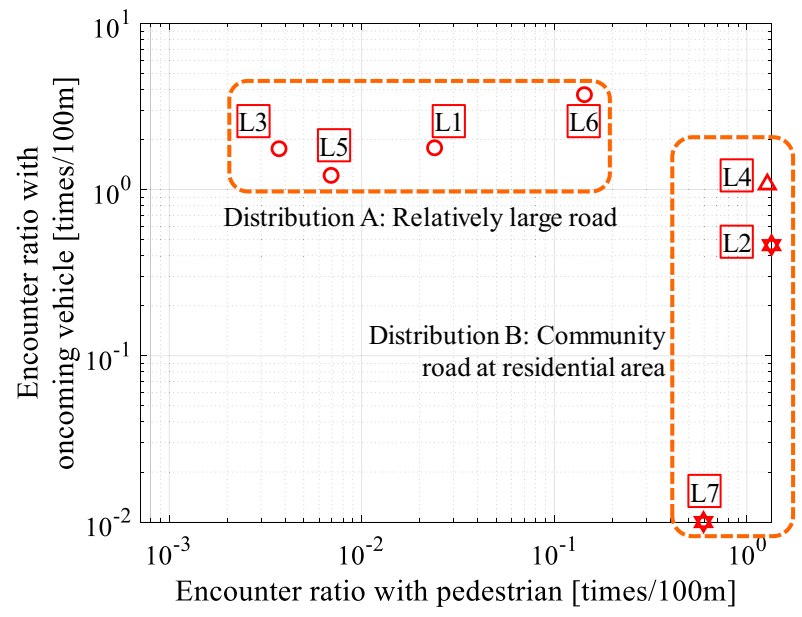

Fig. 16 Encounter ratio with pedestrians and oncoming vehicles in the Koganei area figure, the encounter ratios with the oncoming vehicle at these links are relatively large although those with pedestrians vary. The common point of these links in the real world is that these links are relatively large roads. On the contrary, the Distribution $\mathrm{B}$ shown in the figure includes the remaining results. As shown in the figure, the encounter ratios with the pedestrians at these links are relatively large although those with the oncoming vehicles vary. The common point of these links is that they are community roads at residential areas. Thus, the characteristics of the Koganei area are that it has two kinds of roads and the characteristics of them are clearly different from each other. Therefore, we consider that the drivers in the Koganei area need to adjust their driving modes to adapt to the situations.

Figure 17 shows the encounter ratios in the Kashiwa area. Similar to the Koganei area, we confirm two distributed groups, although the gap between distributions is not as clear as that in the Koganei area. The Distribution A shown in the figure includes the results of L1, L2, L3, L11, and L12, while the Distribution B includes the remaining results. As shown in the figure, the encounter ratios with oncoming vehicle are not as large as those in the Koganei area. The primary different point between distributions in the Kashiwa area is the encounter ratio with the pedestrians. The common point of links in the Distribution $\mathrm{A}$ is the road at non-residential areas while that in the Distribution B is the community road at residential areas. The Kashiwa area characteristics are that the gap between the distribution of the roads at the non-residential area and that at the residential area is relatively small. We consider that the characteristics as a commuter town are reflected in these results.

Figure 18 shows the encounter ratios in the Atsugi area. We confirmed two distributed groups and two links isolated from other links. The Distribution A shown in the figure

: Links where no braking intervention was conducted.

$\triangle$ : Links where braking intervention was conducted at occluded crossings.

$\nabla$ : Links where braking intervention was conducted at stop lines.

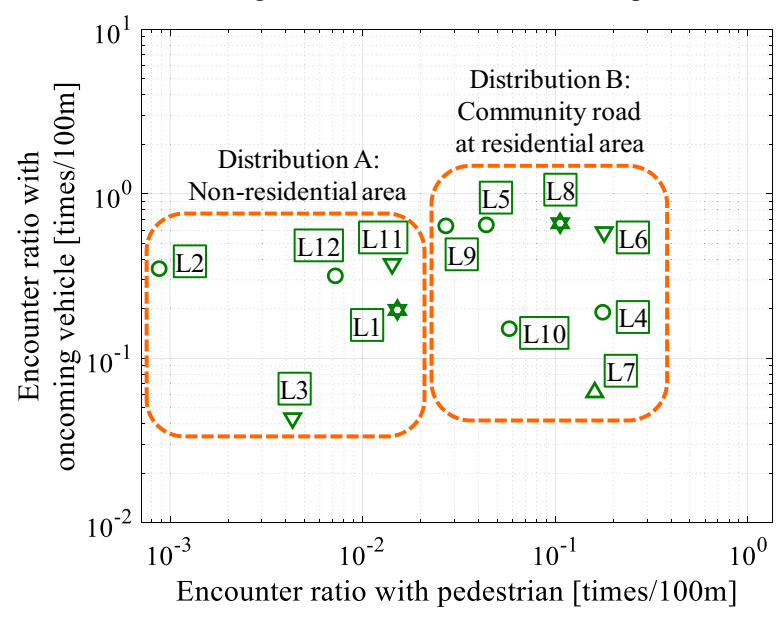

Fig. 17 Encounter ratio with pedestrians and oncoming vehicles in the Kashiwa area 
O: Links where no braking intervention was conducted. $\triangle$ : Links where braking intervention was conducted at occluded crossings. $\nabla$ : Links where braking intervention was conducted at stop lines.

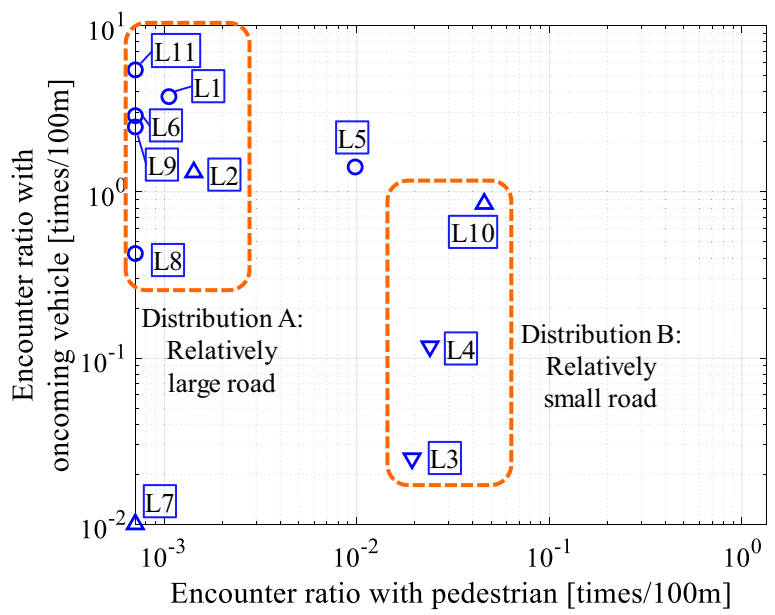

Fig. 18 Encounter ratio with pedestrians and oncoming vehicles in the Atsugi area

includes the results of L1, L2, L6, L8, L9, and L11. As shown in the figure, the encounter ratios with the oncoming vehicle at these links are relatively large while the encounter ratios with pedestrians are small. The common point of these links in the real world is that these links are relatively large roads. On the contrary, the Distribution B includes the results of L3, L4, and L10. As shown in the figure, the encounter ratio with the pedestrians at these links is relatively large in the Atsugi area although it is not as large as those in the other areas. The common point of these links is that these links are relatively small. Actually, L3 and L4 are the roads in paddy fields, and L10 is a minor road. Thus, the characteristics of the Atsugi area is that large roads account for the primary portion. We consider that the characteristics as a provincial city are reflected in these results.

As discussed above, we considered that the driving environmental characteristics were different from each other. Based on these results as a premise, we proceeded with the following analyses.

\subsection{Characteristics of Experimental Participants}

\subsubsection{Evaluation Result of TMT-J}

Each part of the TMT-J classifies the participants into three categories: Normal, border, and abnormal. The criteria are the time to complete the task, and the number of mistakes during the tasks. Tables 8 and 9, which we translated from the original [20], show the criteria for the TMT-J Part A and Part B. Because the TMT-J Part B is more difficult than Part A due to the alternate connection between numbers and characters, a threshold value for allowable number of mistakes are different. As for the threshold values for allowable time, the Brain Function Test Committee of Japan Society for Higher Brain Dysfunction [20] indicates the average time and standard deviation for ages of $60 \mathrm{~s}, 70 \mathrm{~s}$, and $80 \mathrm{~s}$. As for the participants whose age was $60 \mathrm{~s}$, because we recruited elderly drivers whose age was equal to or more than 65 , applying the threshold values for $60 \mathrm{~s}$ to them might result in an extreme underestimation of their ability from a viewpoint of the group. Thus, as representative values, we applied the threshold values for $70 \mathrm{~s}$ to all participants. Therefore, the following analyses discuss to what degree the ability of participants in these experiments were based on a 70 s threshold value. In addition, the Parts A and B results
Table 8 Criteria for TMT-J Part A [20]

\begin{tabular}{lllll}
\hline & Time & & \\
\cline { 3 - 5 } & $\begin{array}{l}\text { Equal to or less than } \\
\text { average + 1SD }\end{array}$ & $\begin{array}{l}\text { Equal to or less than } \\
\text { average +2SD }\end{array}$ & $\begin{array}{l}\text { More than } \\
\text { average +2 SD }\end{array}$ \\
\hline $\begin{array}{c}\text { Number of mis- } \\
\text { takes }\end{array}$ & $\begin{array}{c}\text { Equal to or less } \\
\text { than 2 times } \\
\text { More than 2 } \\
\text { times }\end{array}$ & Border & Border & Abnormal \\
\end{tabular}

\begin{tabular}{lllll}
\hline & Time & & \\
\cline { 3 - 5 } & $\begin{array}{l}\text { Equal to or less than } \\
\text { average +1SD }\end{array}$ & $\begin{array}{l}\text { Equal to or less than } \\
\text { average +2SD }\end{array}$ & $\begin{array}{l}\text { More than } \\
\text { average +2 SD }\end{array}$ \\
\hline $\begin{array}{c}\text { Number of mis- } \\
\text { takes }\end{array}$ & $\begin{array}{c}\text { Equal to or less } \\
\text { than } 4 \text { times } \\
\text { More than } 4 \\
\text { times }\end{array}$ & Bormal & Border & Abnormal \\
\end{tabular}


were independently evaluated. To grasp the general trends, we adopted the worse result in both results. For example, if results of a certain participant were "border" for Part A and "normal" for Part B, we considered the result of the participant as "border."

Table 10 summarizes the evaluation results of the TMTJ. Approximately $80 \%$ of the participants show the normal results. We considered these results as unpaired non-parametric data among the evaluation sites, and conducted statistical analysis. The Kruskal-Wallis test revealed that there are not statistically significant differences between these participants among the sites under a 5\% significance level. Therefore, we considered that there were not so much differences in the participants among the evaluation sites from the ability viewpoints regarding perception and recognition.

\subsubsection{DSQ Evaluation Result}

Figure 19 shows the DSQ evaluation results at each evaluation site. One-way ANOVA revealed that there are statistically significant differences among the average values regarding the "preparatory maneuvers at traffic signals" under a 5\% significance level. In addition, the Tukey-Kramer test revealed that there is a statistically significant difference between the average values of the Koganei area and that of the Atsugi area. To be more precise, the point regarding "preparatory maneuvers at traffic signals" of the participants of the Atsugi area is smaller than that of the Koganei area. On this point, because the participants around the Atsugi area may drive on large roads more frequently than the

Table 10 TMT-J evaluation results

\begin{tabular}{lccc}
\hline & Koganei & Kashiwa & Atsugi \\
\hline Normal & $80.6 \%$ & $75.4 \%$ & $78.6 \%$ \\
Border & $7.5 \%$ & $9.2 \%$ & $14.3 \%$ \\
Abnormal & $11.9 \%$ & $15.4 \%$ & $7.1 \%$ \\
\hline
\end{tabular}

participants in the Koganei area and the interval of traffic signals that the Atsugi area participants experienced daily may be longer than that of the Koganei area; thus, it may lead to this result. For other items, we did not confirm significant differences under a 5\% significance level among the evaluation sites. In this way, the differences in the DSQ evaluation results among the evaluation sites could be interpreted by the effect of differences of driving environmental characteristics.

\subsubsection{WSQ Evaluation Result}

Figure 20 shows the WSQ evaluation results at each evaluation site. One-way ANOVA revealed that there are statistically significant differences among the average values regarding the "tendency to become distracted while driving (DD)", "physical pain/ discomfort (PP)", and "comprehension of driving route (DR)" under a 5\% significance level. As for "DD", the Tukey-Kramer test revealed that there is a statistically significant difference between the average values of the Koganei area and that of the Atsugi area. To be more precise, the workload sensitivity regarding "DD" of the participants of the Koganei area is smaller than that of the Atsugi area. On this point, because the participants in the Koganei area drive their vehicle in an environment where more pedestrians coexist as shown in Fig. 16, they might have more tolerance for driving in a high workload driving environment. As for "PP", the Tukey-Kramer test revealed that there are statistically significant differences between the average values of the Koganei area and the Atsugi area, and also between those of the Kashiwa area and the Atsugi area. To be more precise, the workload sensitivity regarding "PP" of the Atsugi area participants is smaller than that of the Koganei and Kashiwa areas. On this point, because the Atsugi area participants drive the vehicle more frequently than those in the other areas as listed in Table 1, they may have a tolerance regarding the "PP". Moreover, because the average age of the Atsugi area participants is slightly lower than that of the other

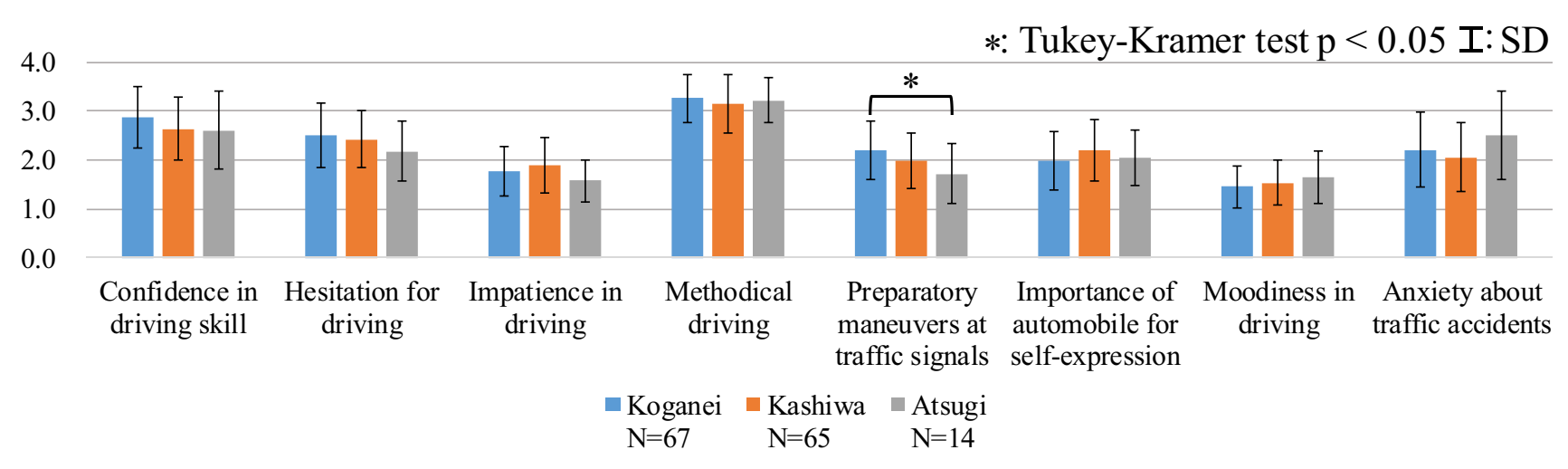

Fig. 19 Comparison of DSQ results 


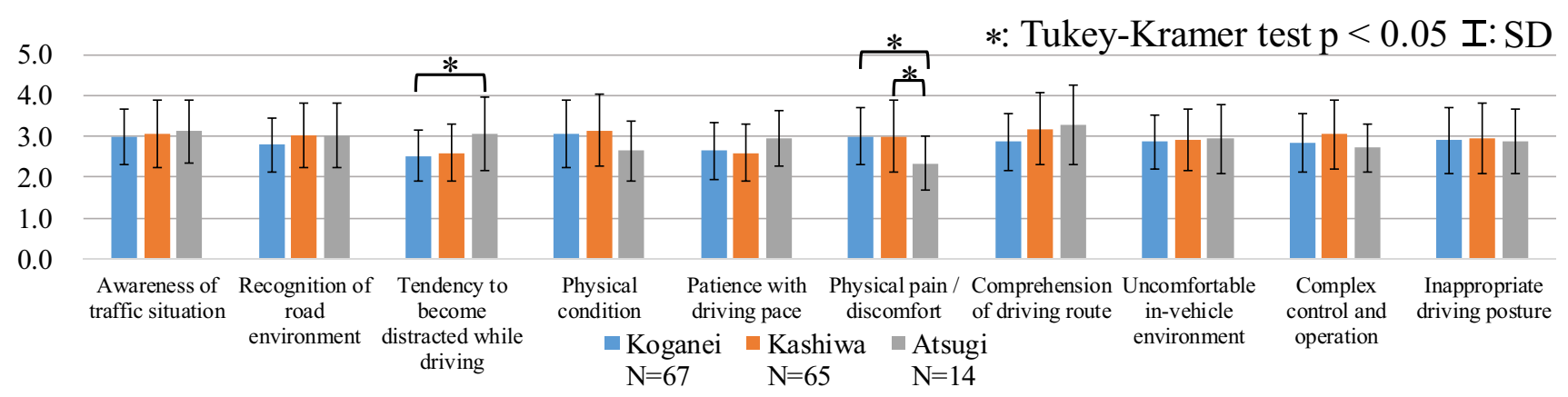

Fig. 20 Comparison of WSQ results

areas as listed in Table 1, that also may affect the result. As for "DR", although One-way ANOVA indicated a significant difference, the Tukey-Kramer test did not reveal any significant difference. On this point, One-way ANOVA might react to the relation between the Koganei and Atsugi area results. Because road networks of the Koganei area is relatively complicated due to many narrow roads, that may affect the result. For other items, we did not confirm significant differences under a 5\% significance level among the evaluation sites. In this way, the large parts of the differences in the WSQ evaluation results among the evaluation sites could be interpreted by the effect of differences of driving environmental characteristics.

\subsection{Comparisons of Acceptability via Questionnaires}

\subsubsection{Evaluation of Reactive Factors}

Figures 21 and 22 show the evaluation results of feeling interference from the support system at occluded crossings and stop lines, respectively. In these cases, "the participants felt very much" means a strong negative evaluation that the participants felt interference from the system. As a general trend, the results of the Koganei, Kashiwa, and Atsugi areas show almost balanced, slightly negative, and slightly positive evaluations, respectively. As for the occluded crossing results, the Kruskal-Wallis test revealed that there are statistically significant differences among the results under a 5\% significance level. In addition, the Steel-Dwass test revealed that there are statistically significant differences between the results of the Kashiwa and Koganei areas, and between those of the Kashiwa and Atsugi areas. As for the stop lines results, the Kruskal-Wallis test revealed the significance differences, and the Steel-Dwass test revealed a significant difference between the results of the Kashiwa and Atsugi areas. On these statistical results, we considered that the experimental conditions might affect the results. The Kashiwa area had a larger number of the crossings and stop lines where the proactive braking interventions were conducted than the other two evaluation sites. In addition, because some of such target situations were located closely to each other, the Kashiwa area
Fig. 21 Comparison of feeling interference from the support system at occluded crossings

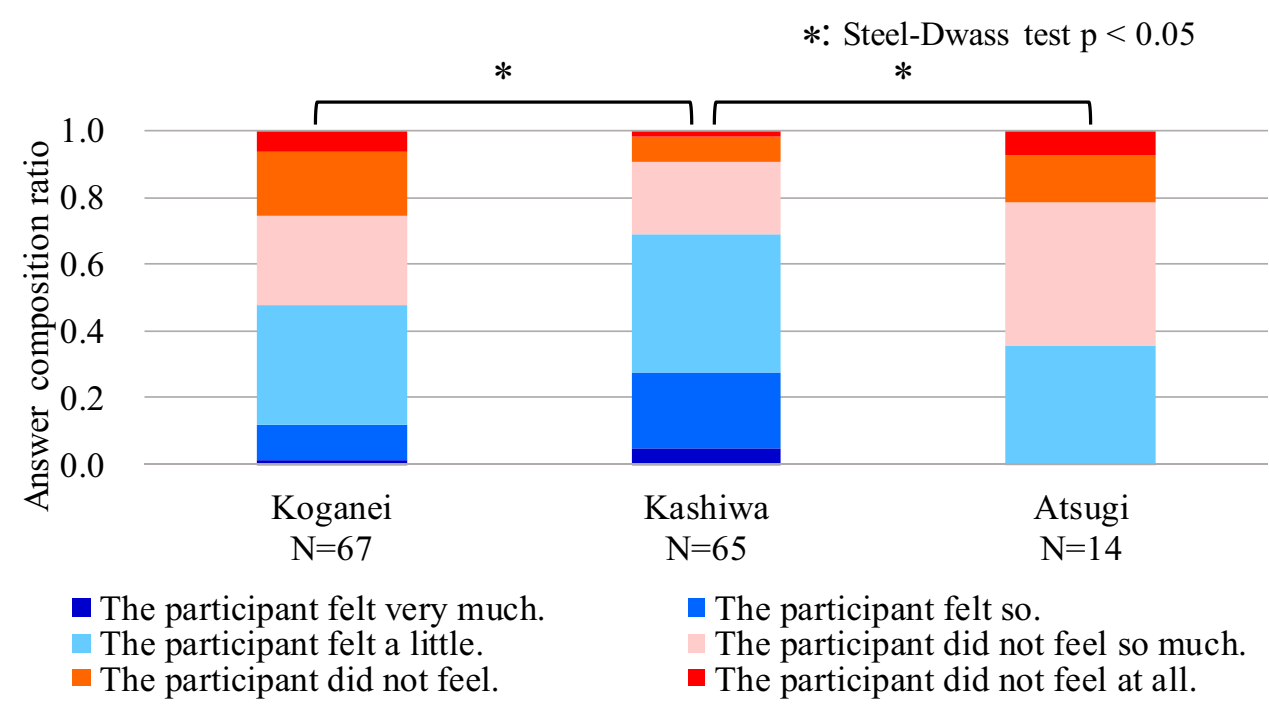

The participant felt a little.

The participant did not feel.
The participant did not feel so much
The participant did not feel at all. 
Fig. 22 Comparison of feeling interference from the support system at stop lines

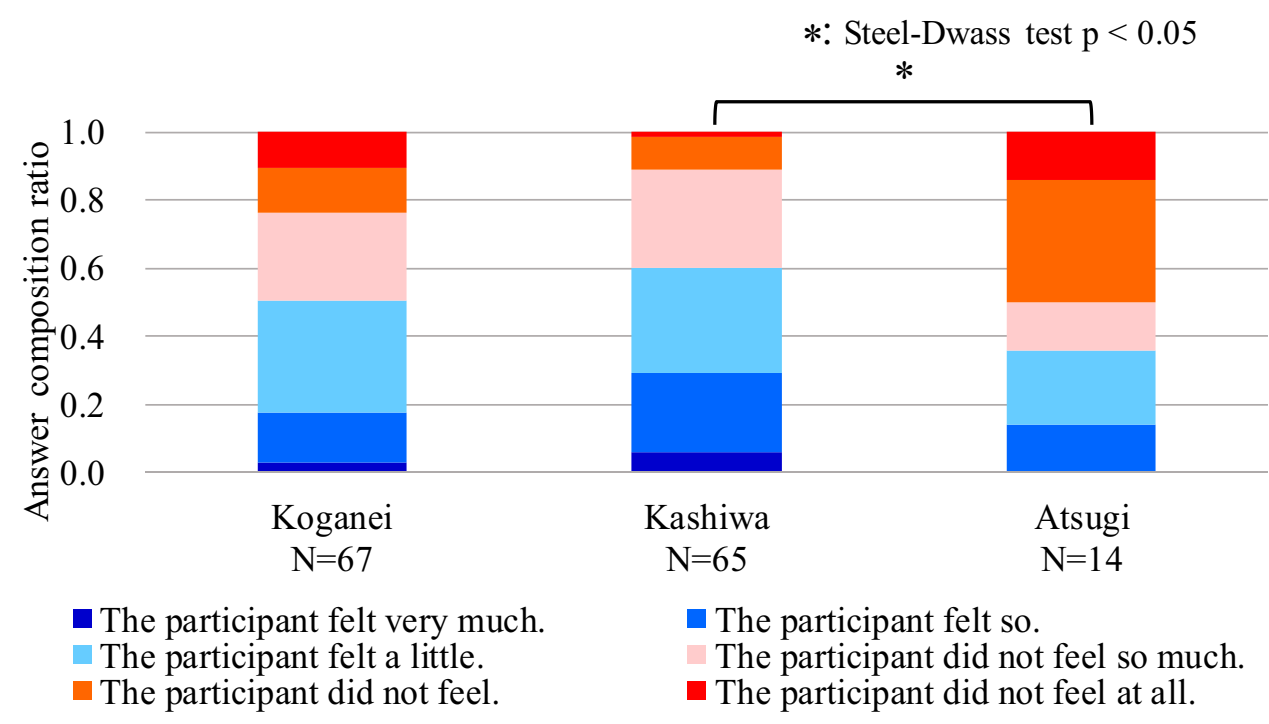

participants experienced braking intervention numerous times in a short period. Thus, the Kashiwa area participants might feel more interference than those of the other evaluation sites. Although the direct reason of these results were experimental conditions, we considered that these experimental conditions were affected indirectly by the urban characteristics. Thus, we considered that these results might depend on the driving environmental characteristics.

Figures 23 and 24 show the evaluation results of feeling anxiety from the support system at occluded crossings and stop lines, respectively. As for the data of the Kashiwa area regarding the stop lines, one participant missed answering the question. Thus, the number of the answers for the Kashiwa area was 64. As a general trend, a large portion of participants at all evaluation sites showed positive evaluations that they did not feel any anxiety from the support system. In addition, the Kruskal-Wallis test revealed that there are not statistically significant differences among the results under a $5 \%$ significance level for both comparisons.
On these results, we considered that the HUD for explaining the proactive braking intervention activation prevented the participants from feeling anxiety.

Figures 25 and 26 show the evaluation results of strangeness feelings from the support system at occluded crossings and stop lines, respectively. Similar to the anxiety case, one Kashiwa area participant missed answering the question regarding the stop lines. Thus, the number of the Kashiwa area answers was also 64. Similar to the feeling interference case, the results of the Koganei, Kashiwa, and Atsugi areas show almost balanced, slightly negative evaluations, and slightly positive evaluations, respectively. As for the occluded crossings, the Kruskal-Wallis test revealed that there are not statistically significant differences among the results under a $5 \%$ significance level. On the contrary, as for the stop lines, although the Kruskal-Wallis test indicates that there are statistically significant differences among the results under a 5\% significance level, the Steel-Dwass test does not reveal any significant differences. On this point, the Kruskal-Wallis test
Fig. 23 Comparison of feeling anxiety for the support system at occluded crossings

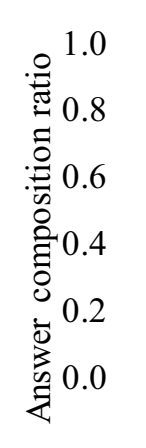

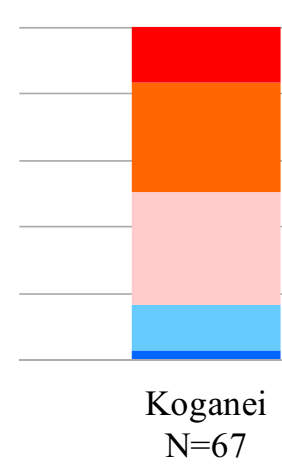

The participant felt very much. The participant felt a little.

The participant did not feel.

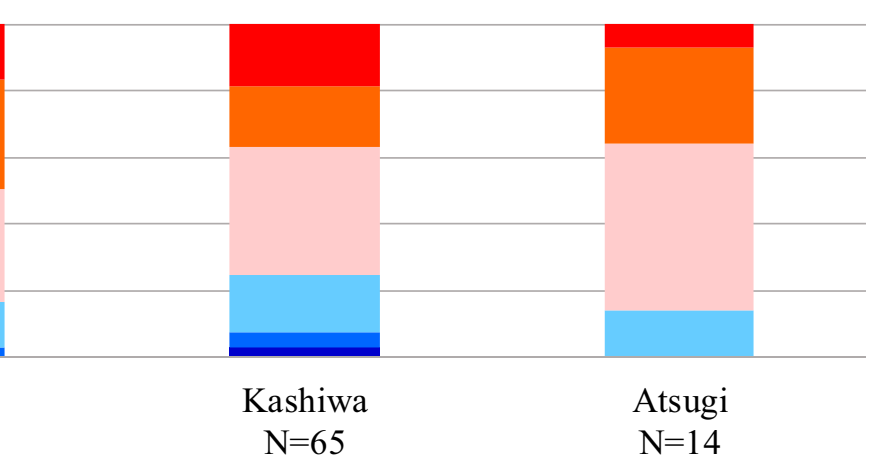

- The participant felt so.

The participant did not feel so much. - The participant did not feel at all. 
Fig. 24 Comparison of feeling anxiety for the support system at stop lines
Fig. 25 Comparison of strangeness feeling for the support system at occluded crossings
Fig. 26 Comparison of strangeness feeling for the support system at stop lines

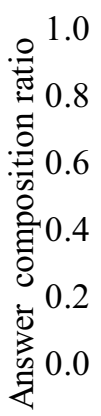

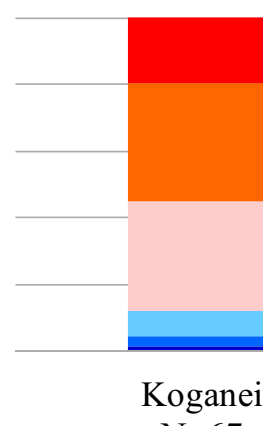

$$
\mathrm{N}=67
$$

The participant felt very much

The participant felt a little.

- The participant did not feel.

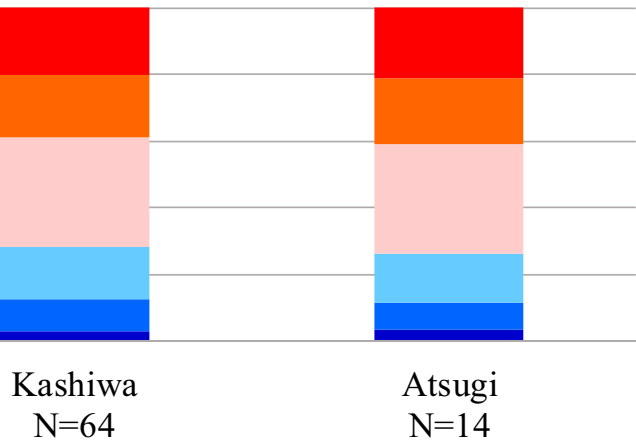

- The participant felt so.

The participant did not feel so much.

- The participant did not feel at all.

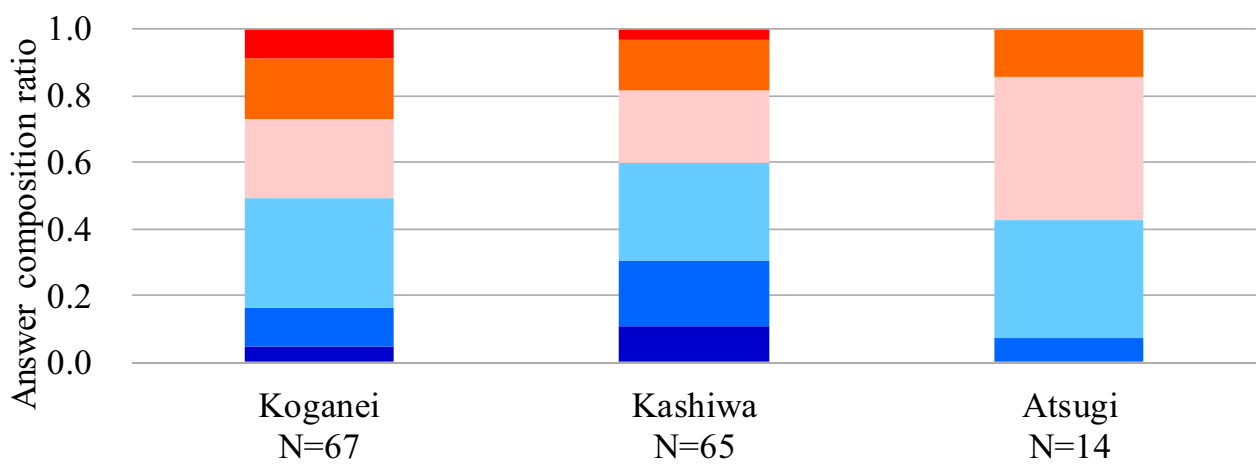

- The participant felt very much.

The participant felt a little.

- The participant did not feel.
- The participant felt so.

The participant did not feel so much.

- The participant did not feel at all.

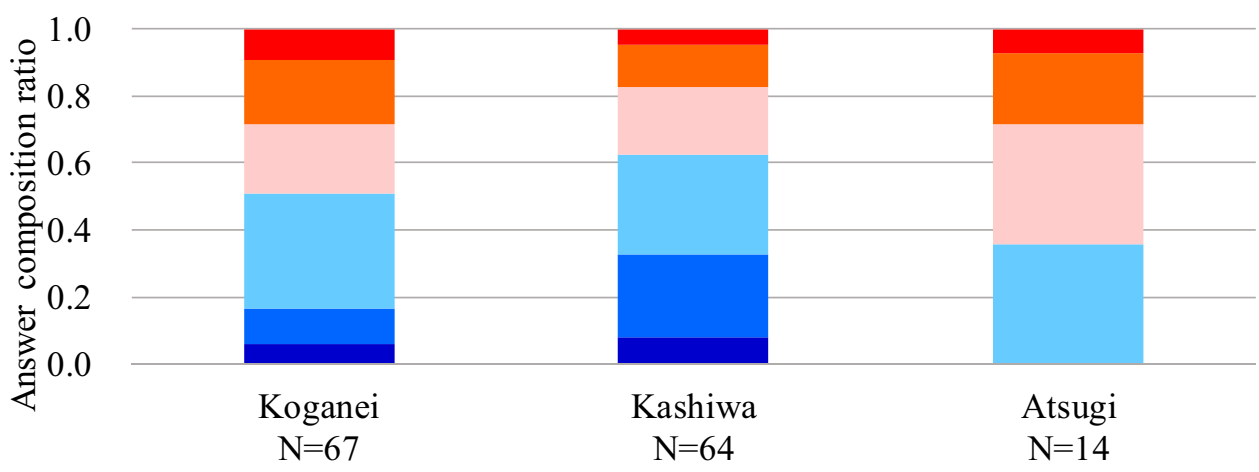

The participant felt very much.

The participant felt a little.

The participant did not feel.
- The participant felt so.

The participant did not feel so much.

- The participant did not feel at all. 
may react to the relation between the Kashiwa and Atsugi area results. Regarding the differences of the balance between the positive and negative evaluations for the feeling of strangeness among the evaluations sites, we considered that the characteristics of driving environments might affect the evaluation results. As for the stop lines S1 and S2 of the Koganei area, and $\mathrm{S} 2$ of the Atsugi area, the stop lines were located around the intersections where the road priority was evincive due to the differences of the road width. On the other hand, because some target stop lines of the Kashiwa area were located on community roads, the road priority around them at Kashiwa area was not clear; further, such an unclear priority might result in lack of feeling for the necessity to stop at the crossings. Thus, we considered that such situations might result in more feeling of strangeness at the Kashiwa area than at other evaluation areas. In this way, similar to the feeling interference case, we considered that these results might depend on the driving environmental characteristics.

\subsubsection{Evaluation of Comprehensive Factors}

Figure 27 shows the evaluation results of a driving burden reduction feeling owing to the support system. In these cases, "the participants felt very much" means a strong positive evaluation that the participants could reduce the driving burden owing to the system. As a general trend, a large portion of the participants at all evaluation sites answered that they felt a burden reduction owing to the support system. As for the statistical analyses, although the Kruskal-Wallis test indicates that there are statistically significant differences among the results under a 5\% significance level, the Steel-Dwass test does not reveal any significant differences. The Kruskal-Wallis test may react to the relation between the Koganei and Kashiwa area results. On this point, we consider that the driving difficulties might affect the evaluation results. As discussed in Sections 4.1 and 4.2, because there were many coexisting traffic participants on the Koganei area road, the driving workload might be higher than other evaluation sites. Thus, although the proposed system could reduce the burden related to pedestrians rushing from occluded areas, there may still be other difficulties while driving in the Koganei area unlike other evaluation sites. We consider that such differences might be the reason for the abovementioned statistical results. However, although the Kruskal-Wallis test revealed statistically significant differences among the evaluations results, the evaluation results are not balanced, consisting of both positive and negative evaluations, but as a whole positive. Therefore, we consider that the proposed system was positively evaluated from this viewpoint.

Figure 28 shows the evaluation results of a safe driving feeling owing to the support system. Similar to the burden reduction feeling, a large portion of the participants at all evaluation sites answered that they could drive more safely owing to the support system. The Kruskal-Wallis test reveals that there are not statistically significant differences among the results under a 5\% significance level. From these results, we considered that the participants felt benefits from the proposed system.

\subsubsection{Evaluation of Reflective Factors}

Figures 29, 30, and 31 show the evaluation results of the support system necessity for elderly drivers in Japanese society, for themselves currently, and for themselves after 5 years, respectively. As a general trend, the evaluation differences among the questions seem larger than those among the evaluation sites for the same question. Actually, for each comparison of the same question, the Kruskal-Wallis test does not indicate significant differences among the results under a 5\% significance level. Thus, on these points, we considered that there were not differences among the evaluation sites. Therefore, we considered that the participants of each evaluation site were equivalent regarding these factors, and compared the answers between the questions. Figure 32 shows the comparison results of answers regarding a feeling
Fig. 27 Comparison of driving burden reduction feeling owing to the support system

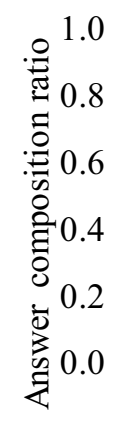

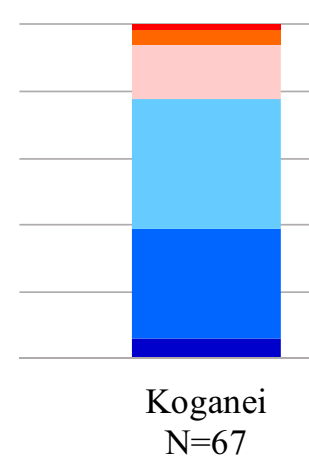

The participant felt very much. The participant felt a little. The participant did not feel.

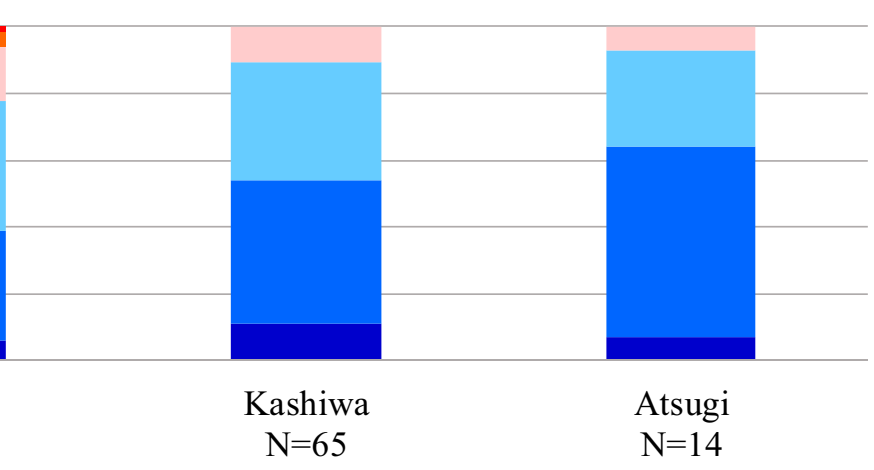

The participant felt so.

The participant did not feel so much. The participant did not feel at all. 
Fig. 28 Comparison of safe driving feeling owing to the support system
Fig. 29 Comparison of feeling necessity for the support system for elderly drivers in Japanese society
Fig. 30 Comparison of feeling necessity for the support system for themselves currently

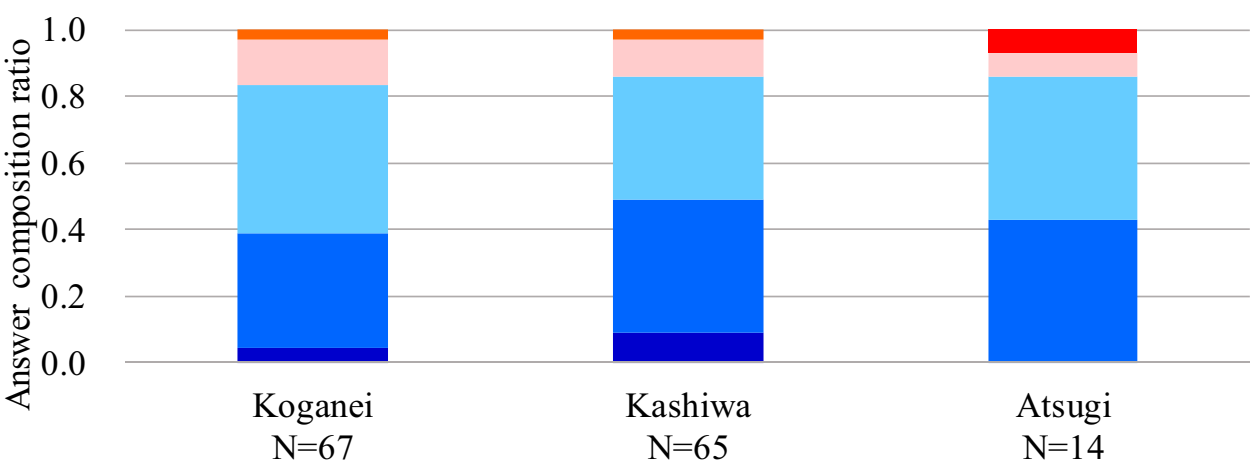

- The participant felt very much.

The participant felt a little.

- The participant did not feel.

The participant felt so.

The participant did not feel so much.

- The participant did not feel at all.

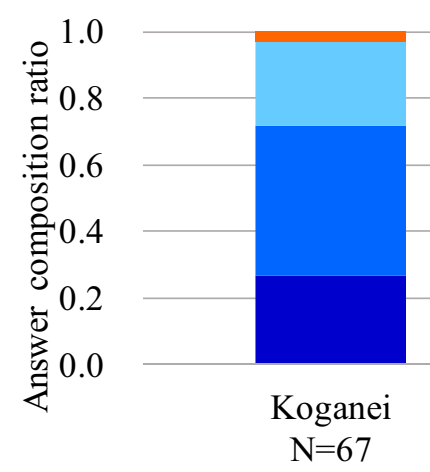

- The participant felt very much.

The participant felt a little.

The participant did not feel.

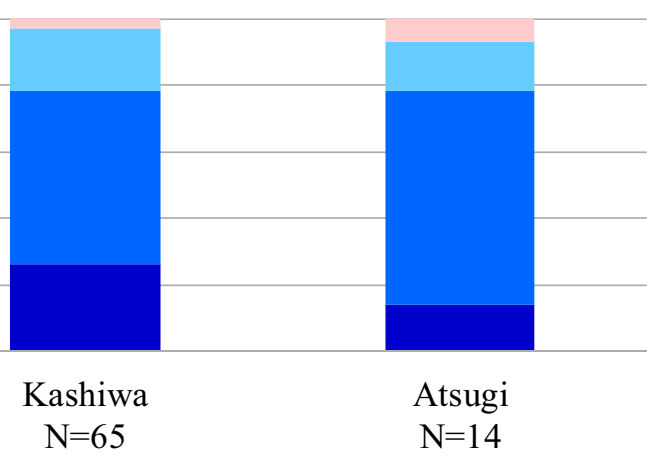

The participant felt so.

The participant did not feel so much.

- The participant did not feel at all.

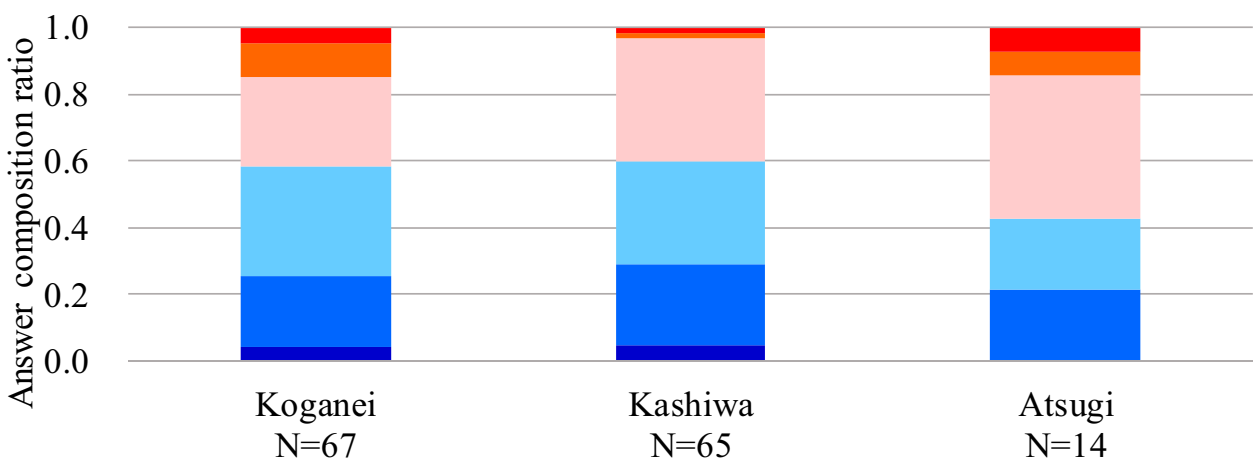

- The participant felt very much.

The participant felt a little.

- The participant did not feel.
The participant felt so.

The participant did not feel so much.

- The participant did not feel at all. of necessity. The total number of the answers for each question is 146. The Friedman's test indicates that there are statistically significant differences among the answers under a 5\% significance level. In addition, the Steel-Dwass test reveals significant difference between the answers regarding elderly drivers in Japanese society and those regarding themselves 
Fig. 31 Comparison of feeling necessity for the support system for themselves after five years
Fig. 32 Comparison of answers regarding the feeling of necessity

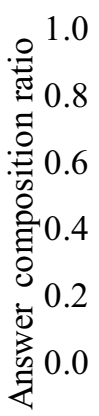
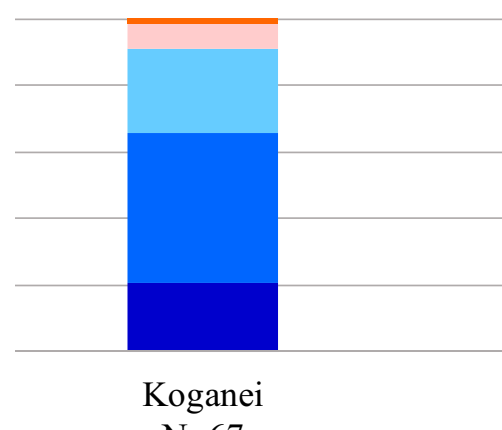

$$
\begin{gathered}
\text { Koganei } \\
\mathrm{N}=67
\end{gathered}
$$

\section{Kashiwa \\ $\mathrm{N}=65$}

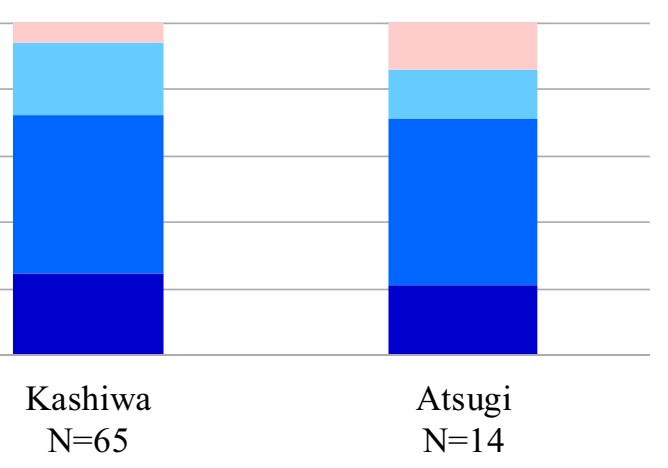

- The participant felt so.

The participant did not feel so much.

- The participant did not feel at all.
Atsugi $\mathrm{N}=14$

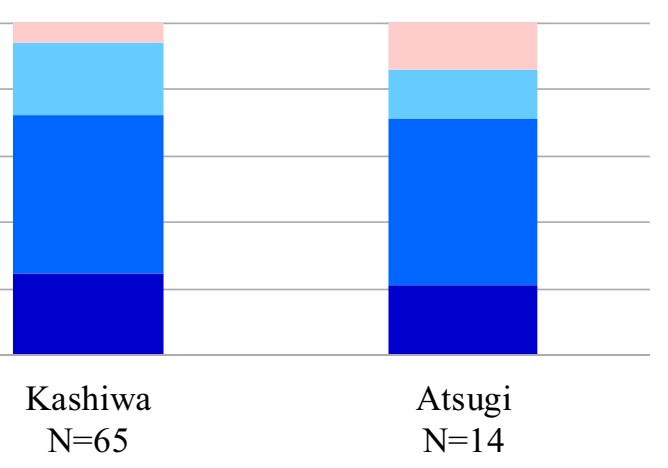

The participant felt a little.

- The participant did not feel.

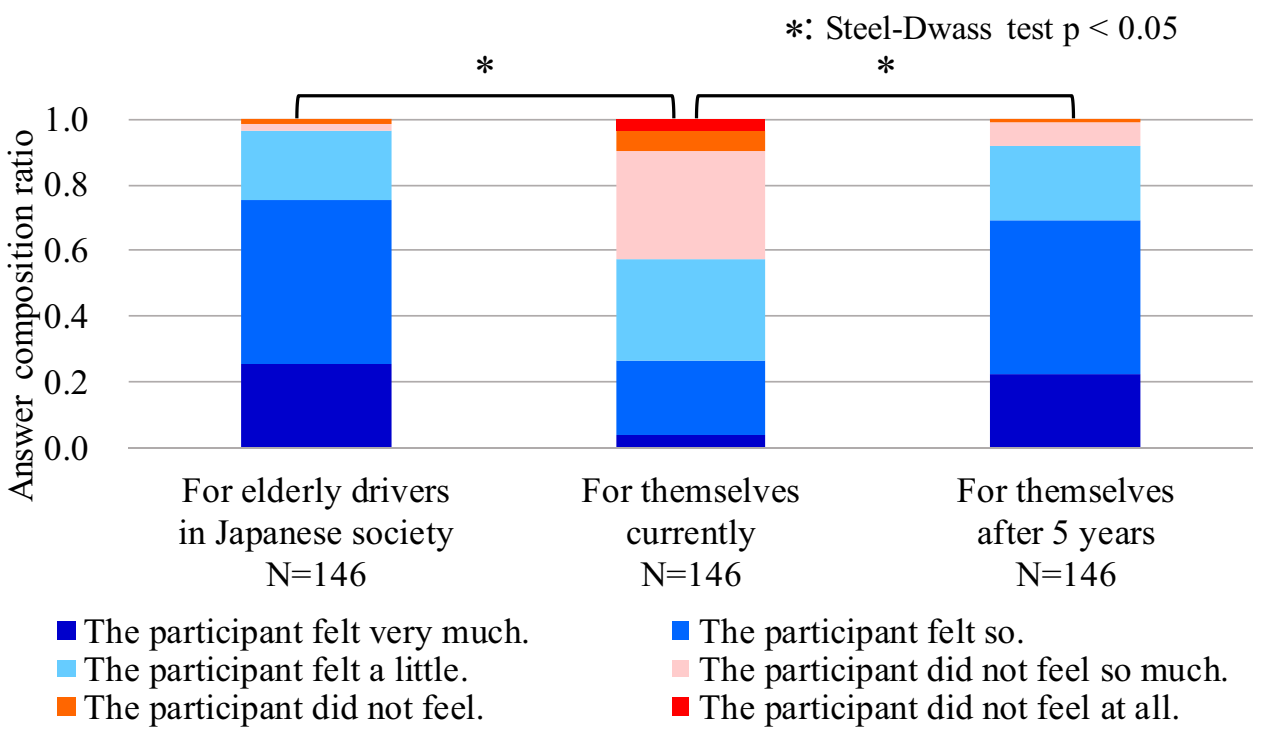

currently, and between those regarding the themselves currently and those regarding themselves after 5 years. These results indicate the following trends.

- A large portion of participants considered that they had better driving abilities than other elderly drivers in Japanese society.

- A large portion of participants was conscious that their driving abilities would decline due to their further aging, at least within the next 5 years.

- A large portion of participants considered that the proposed system would compensate such driving ability declines.

- Therefore, a large portion of participants considered that the necessity for the proposed system would increase.

\subsubsection{Evaluation of Desire to Introduce the system}

Figure 33 shows the evaluation results of a desire to introduce the support system into their own vehicle. In these cases, "the participants felt very much" means a strong positive evaluation that the participants want to introduce the proposed system into their own vehicle. As a general trend, more than half of the participants at all evaluation sites answered that they want to introduce the proposed system into their own vehicle. The Kruskal-Wallis test reveals that there are not statistically significant differences among the results under a $5 \%$ significance level.

\subsection{Discussions Based on Summary of Comparisons Among Evaluations Sites}

As discussed in Section 2.4, the primary intention of this study was the discussion regarding the generalities of acceptability of 
Fig. 33 Comparison of a desire to introduce the support system into their own vehicle

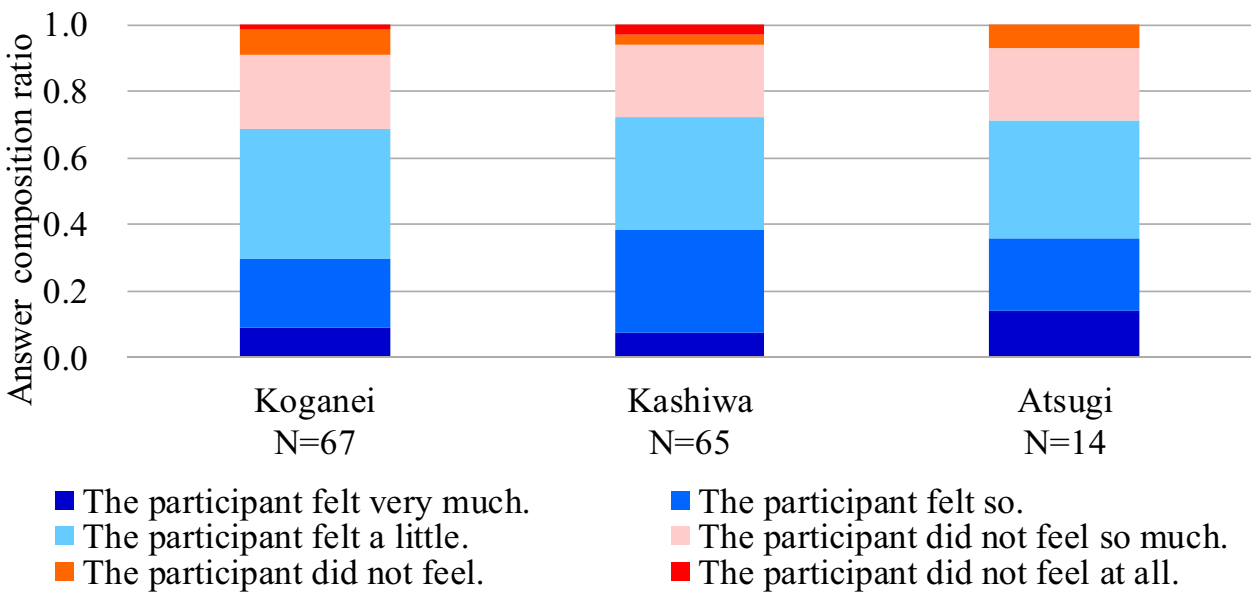

previous study [18], we constructed the prototype system of adaptive proactive braking intervention based on a risk map, and confirmed the effectiveness for improving the evaluations regarding the reactive factors to a certain degree.

- Adaptation of $\mathrm{V}_{\text {min }}$ based on the road characteristics.

- Development of adaptive information provision devices explaining the detailed situations regarding the cross roads and intervention reasons.

Conversely, we did not confirm any statistically significant differences among the evaluation sites for the comprehensive factors, reflective factors, and total acceptability. Thus, we consider that the proposed system main concept was positively evaluated in common. In short, we confirmed the generalities of the acceptability regarding the main concept of the proposed system at least in the range of this study.

\section{Conclusions}

To discuss the generalities of the acceptability of the proactive braking intervention system, we conducted field operation tests, where a total of 146 elderly drives participated at three evaluations sites. The collected FOT data analyses showed the following though these results were limited in the characteristics of driving environment discussed in this study.

- The driving environmental characteristics were different from each other among the evaluation sites.

- The driver characteristics of experimental participants were considered almost equivalent among the sites except in terms of the effect of driving environmental characteristics.

- We considered that reactive factors of the acceptability depended on the driving environmental characteristics 
Table 11 Summary of comparison results among the evaluation sites

\begin{tabular}{|c|c|c|}
\hline Topic & $\begin{array}{l}\text { Statistical differences under a } 5 \% \text { significance } \\
\text { level among the sites } \\
\text { KW: Kruskal-Wallis } \\
\text { SD: Steel-Dwass }\end{array}$ & Abstract of comparison results \\
\hline \multicolumn{3}{|l|}{ Reactive factors } \\
\hline Interference at crossings & $\begin{array}{l}\text { Significant among the regions by KW test } \\
\text { Significant between Kashiwa and Koganei by } \\
\text { SD test } \\
\text { Significant between Kashiwa and Atsugi by } \\
\text { SD test }\end{array}$ & $\begin{array}{l}\text { Results at Kashiwa show slightly negative results } \\
\text { Results at Koganei show almost balanced results } \\
\text { Results at Atsugi show slightly positive results }\end{array}$ \\
\hline Interference at stop lines & $\begin{array}{l}\text { Significant among the regions by KW test } \\
\text { Significant between Kashiwa and Atsugi by } \\
\text { SD test }\end{array}$ & $\begin{array}{l}\text { Results at Kashiwa show slightly negative results } \\
\text { Results at Koganei show almost balanced results } \\
\text { Results at Atsugi show slightly positive results }\end{array}$ \\
\hline Anxiety at crossings & Not significant & More than half evaluations show positive answers \\
\hline Anxiety at stop lines & Not significant & $\begin{array}{l}\text { More than half of the evaluations show positive } \\
\text { answers }\end{array}$ \\
\hline Strangeness at crossings & Not significant & $\begin{array}{l}\text { Results at Kashiwa show slightly negative results } \\
\text { Results at Koganei show almost balanced results } \\
\text { Results at Atsugi show slightly positive results }\end{array}$ \\
\hline Strangeness at stop lines & $\begin{array}{l}\text { Significant among the regions by } \mathrm{KW} \text { test } \\
\text { Not significant by SD test }\end{array}$ & $\begin{array}{l}\text { Results at Kashiwa show slightly negative results } \\
\text { Results at Koganei show almost balanced results } \\
\text { Results at Atsugi show slightly positive results }\end{array}$ \\
\hline \multicolumn{3}{|l|}{ Comprehensive factors } \\
\hline Reduction of burden & $\begin{array}{l}\text { Significant among the regions by KW test } \\
\text { Not significant by SD test }\end{array}$ & $\begin{array}{l}\text { More than half of the evaluations show positive } \\
\text { answers }\end{array}$ \\
\hline Safe driving & Not significant & $\begin{array}{l}\text { More than half of the evaluations show positive } \\
\text { answers }\end{array}$ \\
\hline \multicolumn{3}{|l|}{ Reflective factors } \\
\hline Necessity for elderly drivers in the society & Not significant & $\begin{array}{l}\text { More than half of the evaluations show positive } \\
\text { answers }\end{array}$ \\
\hline Necessity for themselves currently & Not significant & $\begin{array}{l}\text { Results at Koganei and Kashiwa show slightly } \\
\text { positive results } \\
\text { Results at Atsugi show slightly negative results }\end{array}$ \\
\hline Necessity for themselves after five years & Not significant & $\begin{array}{l}\text { More than half of the evaluations show positive } \\
\text { answers }\end{array}$ \\
\hline \multicolumn{3}{|l|}{ Total evaluation } \\
\hline Desire to introduce the system & Not significant & $\begin{array}{l}\text { More than half of the evaluations show positive } \\
\text { answers }\end{array}$ \\
\hline
\end{tabular}

because there were statistically significant differences regarding some of the reactive factors among the sites.

- We confirmed the generalities that the main concept of the proposed system was positively evaluated independently of the driving environmental characteristics because there were not statistically significant differences regarding other factors.

In the future plan, we will analyze the behavioral details of individual participants and interactions with the proposed system from the time-series data. In addition, analysis regarding driving-behavior changes of the participants between before and after experiencing the proposed system is another necessary discussion.
Acknowledgements This research has been conducted as a part of the research project "Autonomous Driving System to Enhance Safe and Secured Traffic Society for Elderly Drivers" granted by Japan Science and Technology Agency(JST), Strategic Promotion of Innovative Research and Development.

Open Access This article is licensed under a Creative Commons Attribution 4.0 International License, which permits use, sharing, adaptation, distribution and reproduction in any medium or format, as long as you give appropriate credit to the original author(s) and the source, provide a link to the Creative Commons licence, and indicate if changes were made. The images or other third party material in this article are included in the article's Creative Commons licence, unless indicated otherwise in a credit line to the material. If material is not included in the article's Creative Commons licence and your intended use is not permitted by statutory regulation or exceeds the permitted use, you will need to obtain permission directly from the copyright holder. To view a copy of this licence, visit http://creativecommons.org/licenses/by/4.0/. 


\section{References}

1. Cabinet Office, Annual Report on the Aging Society [Summary] FY 2019. https://www8.cao.go.jp/kourei/english/annualreport/ 2019/pdf/2019.pdf. Accessed 17 Apr 2020

2. Cabinet Office, FY 2018 Situation of Road Traffic Accidents and Current State of Traffic Safety Measures, http://www8.cao.go.jp/ koutu/taisaku/h29kou_haku/english/wp2017-pdf.html. Accessed 17 Apr 2020

3. Inoue, H., Raksincharoensak, P., Inoue, S.: Intelligent driving system for safer automobiles. Journal of Information Processing 25, 32-43 (2017)

4. Akamatsu, M.: Handbook of Automotive Human Factors, pp. 17-18. CRC Press, Boca Raton (2019)

5. Institute for Traffic Accident Research and Data Analysis, ITARDA INFORMATION, No. 119 (2016), (accessed on 17 April, 2020). (in Japanese)

6. Masao, F.: The latest trend of $\mathrm{v} 2 \mathrm{x}$ driver assistance systems in Japan. Comput. Netw. 55, 3134-3141 (2011)

7. Saito, Y., Raksincharoensak, P.: Shared control in risk predictive braking maneuver for preventing collisions with pedestrians. IEEE Transactions on Intelligent Vehicles 1(4), 314-324 (2016)

8. Ito, T., Mio, M., Tohriyama, K., Kamata, M.: Novel map platform based on primitive elements of traffic environments for automated driving technologies. International Journal of Automotive Engineering 7(4), 143-151 (2016)

9. Saito, Y., Yoshimi, R., Kume, S., Imai, M., Yamasaki, A., Ito, T., Inoue, S., Shimizu, T., Nagai, M., Inoue, H., Raksincharoensak, P.: Effects of a driver assistance system with foresighted deceleration control on the driving performance of elderly and younger drivers. Transport. Res. F: Traffic Psychol. Behav. 77, 221-235 (2021)

10. Ito, T., Shino, T., Kamata, M.: Information sharing to improve understanding of proactive braking intervention for elderly drivers. Int. J. Intell. Transp. Syst. Res. 16(3), 173-186 (2018)

11. Ito, T., Shino, T., Kamata, M.: Information Sharing to Improve Understanding of Proactive Steering Intervention for Elderly Drivers. Int. J. Intell. Transp. Syst. Res. 17(1), 18-31 (2019)

12. Ito, T., Shino, T., Kamata, M.: Effectiveness of Information Sharing to Improve Elderly Drivers' Acceptability for Proactive Intervention Systems. International journal of automotive engineering 10(1), 55-64 (2019)

13. Kessler, C., Etemad, A., Alessandretti, G., Heinig, K., Selpi, R., Brouwer, A., Cserpinszky, W., Hagleitner, M. Benmimoun, SP1 D11.3 Final Report. https://www.eurofot-ip.eu/download/libra ry/deliverables/eurofotsp120121212v11dld113_final_report. pdf. Accessed 21 Apr 2020

14. Ito, T., Shino, T., Kamata, M.: Initial investigation of elderly drivers' acceptability for proactive intervention by intelligent vehicle. Int. J. Intell. Transp. Syst. Res. 16(1), 51-65 (2018)

15. Inoue, S., Saito, Y., Yamasaki, A., Kinoshita, T., Sato, F., Ito, T., Shimizu, T., Saito, S., Uchida, N., Inoue, H., Pongsathorn, R.: Risk Predictive Steering Driver Assistance System Based on Shared Ratio between Human and Machine. Transactions of Society of Automotive Engineers of Japan 50(6), 1646-1652 (2019). (in Japanese)

16. Ito, T., Soya, M., Nakamura, S., Saito, S., Uchida, N., Kamata, M.: Acceptability of a Proactive Braking Intervention System by Elderly Drivers Using an Actual Vehicle. International Journal of Automotive Engineering 9(4), 186-194 (2018)
17. Matsumi, R., Oya, K., Ito, T., Saito, Y., Mio, M., Hashimoto, N., Nagai, M., Inoue, H., Kamata, M.: Acceptability Investigation of Proactive Braking Intervention for Elderly Drivers on Community Roads. Transactions of Society of Automotive Engineers of Japan 50(3), 911-917 (2019). (in Japanese)

18. Ito, T., Soya, M., Tohriyama, K., Saito, Y., Shimizu, T., Yamasaki, A., Nagai, M., Inoue, H., Kamata, M.: Evaluation of Acceptability of Adaptive Proactive Braking Intervention System Based on Risk Map for Elderly Drivers. International Journal of Automotive Engineering 11(2), 40-48 (2020)

19. Geospatial Information Authority of Japan: GSI Maps (online), https://maps.gsi.go.jp/, (accessed on 2020-03-22).

20. Brain Function Test Committee of Japan Society for Higher Brain Dysfunction, Trail Making Test Japanese edition (TMTJ), Shinkoh Igaku Shuppan Co. Ltd., (2019). (in Japanese)

21. Armitage, S.G.: An analysis of certain psychological tests used for the evaluation of brain injury. Psychol. Monogr. 60(1), 1-48 (1946)

22. Ishibashi, M., Okuwa, M., Doi, S., Akamatsu, M.: Indices for Characterizing Driving Style and their Relevance to Car Following Behavior. Proceedings of SICE Annual Conference 2007, 1132-1137 (2007)

23. Ishibashi, M., Okuwa, M., Doi, S., Akamatsu, M.: Characterizing Indices of Driver's Workload Sensitivity and their Relevance to Route Choice Preferences. Transactions of Society of Automotive Engineers of Japan 39(5), 169-174 (2008). (in Japanese)

Publisher's Note Springer Nature remains neutral with regard to jurisdictional claims in published maps and institutional affiliations.

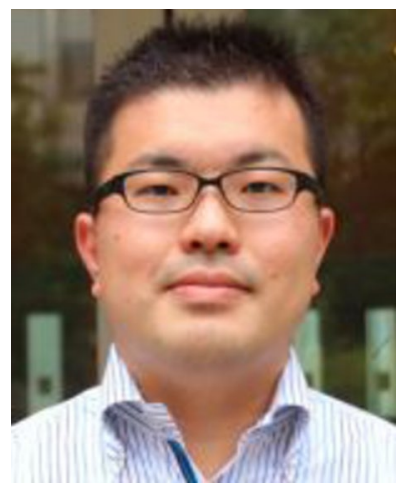

Takuma Ito Takuma Ito is a project lecturer at Department of Mechanical Engineering, The University of Tokyo, Japan. He received his Doctor of Engineering degree from The University of Tokyo in 2012. His research interests are autonomous vehicles and human machine interactions.

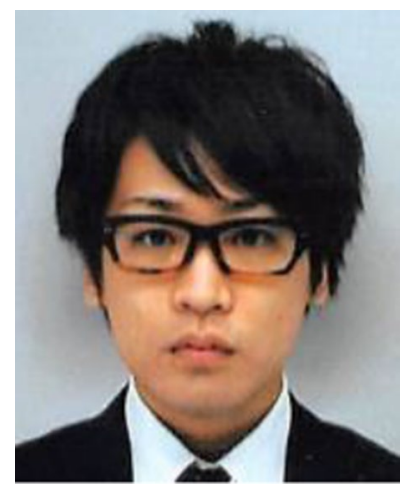

Ryosuke Matsumi Ryosuke Matsumi is a project researcher at Institute of Gerontology, The University of Tokyo, Japan. He received his Doctor of Engineering degree from Tokyo University of Agriculture and Technology in 2014. 


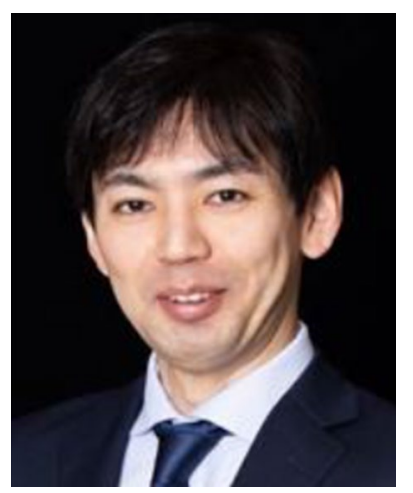

Yuichi Saito Yuichi Saito is an Assistant Professor at University of Tsukuba. He received his Doctor of Engineering degree from University of Tsukuba in 2015. His research interests are smart collaborations between human and machine, shared control, and vehicle dynamics.

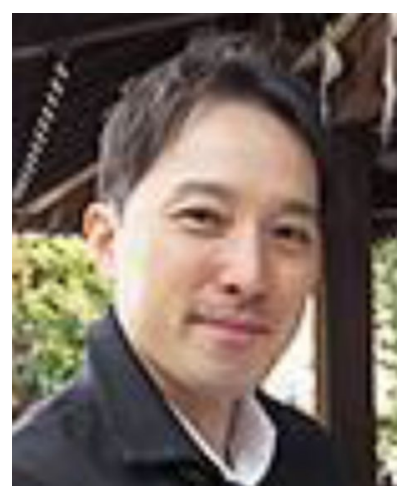

Akito Yamasaki Akito Yamasaki is an Assistant Professor at Meijo University. He received his Ph. D degree in Systems Science and Informatics from Hokkaido University in 2010. His research interests are computer vision, human behavior imaging and autonomous vision.

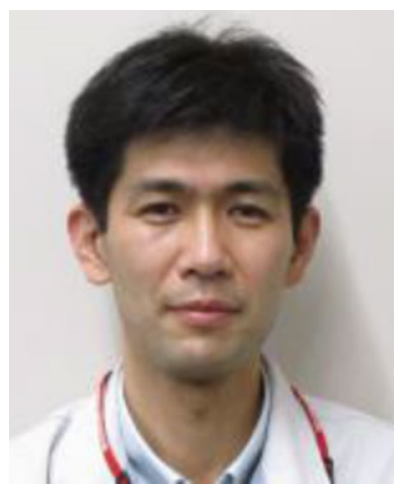

Shintaro Inoue Shintaro Inoue is a group manager of Automated Driving \& Advanced Safety System Development Div. at Toyota Motor Corporation in Japan. He received his Doctor of Engineering degree from Tokyo University of Agriculture and Technology in 2007. His research interests are vehicle dynamics and motion planning of an automated vehicle.

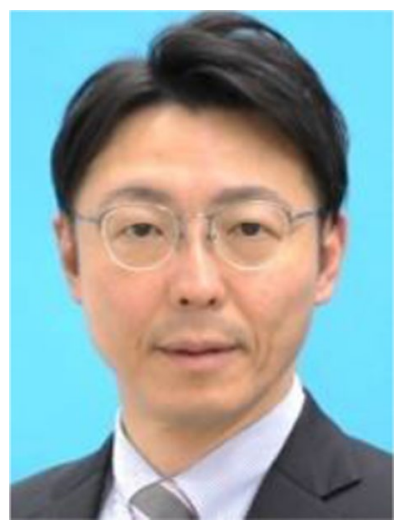

Tsukasa Shimizu Tsukasa Shimizu is a program manager at TOYOTA CENTRAL R\&D LABS., INC. He received his Master of Human and Environmental Studies degree from Kyoto University in 1996. His research interests are ADAS and Automated Driving.

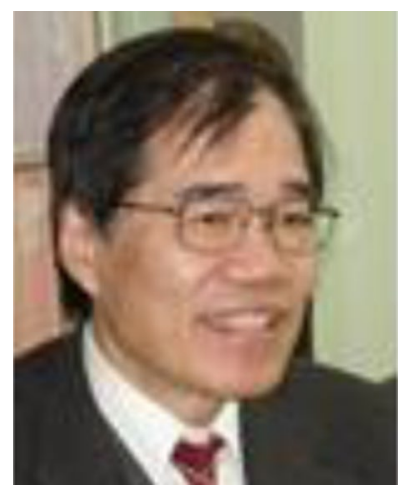

Masao Nagai Since 2013, Masao Nagai is a president of Japan Automobile Research Institute, and a Professor emeritus of Tokyo University of Agriculture and Technology. He received his Doctor of Engineering degree from The University of Tokyo in 1977. His research interests are advanced vehicle dynamics and control, active safety, ADAS and Automated Driving.

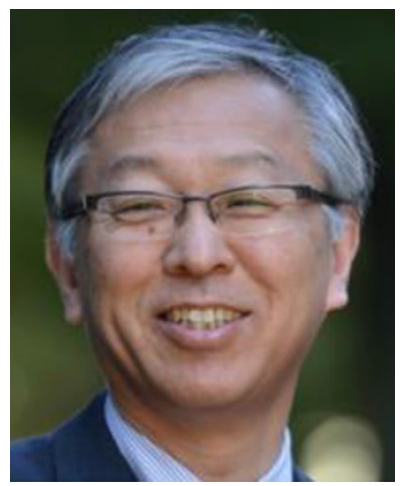

Hideo Inoue Hideo Inoue is a Professor at Kanagawa Institute of Technology and a visiting Professor at Tokyo University of Agriculture and Technology in Japan. He received Bachelor of Mechanical Engineering degree from Waseda University in 1978. His research interests are advanced driver assistance systems and automated driving systems for enhancing safety, and driving dynamics control.

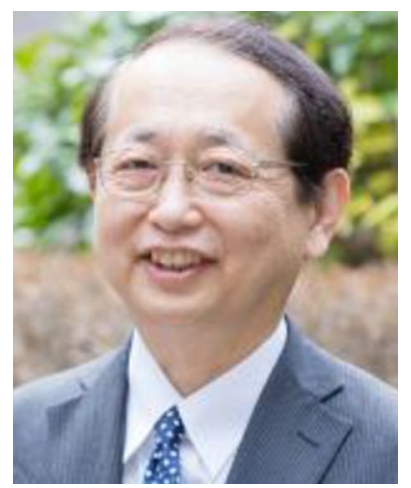

Minoru Kamata Minoru Kamata is a professor at The University of Tokyo in Japan. He received his Doctor of Engineering degree from The University of Tokyo in 1987 in the field of mechanical engineering. His research interests are advanced driver assistance systems for enhancing active safety and assistive technology for elderly and disabled. 